\title{
A THEORY OF DISAGREEMENT IN REPEATED GAMES WITH BARGAINING
}

\begin{abstract}
By DAVID A. Miller AND JOEL WATSON ${ }^{1}$
This paper proposes a new approach to equilibrium selection in repeated games with transfers, supposing that in each period the players bargain over how to play. Although the bargaining phase is cheap talk (following a generalized alternating-offer protocol), sharp predictions arise from three axioms. Two axioms allow the players to meaningfully discuss whether to deviate from their plan; the third embodies a "theory of disagreement" - that play under disagreement should not vary with the manner in which bargaining broke down. Equilibria that satisfy these axioms exist for all discount factors and are simple to construct; all equilibria generate the same welfare. Optimal play under agreement generally requires suboptimal play under disagreement. Whether patient players attain efficiency depends on both the stage game and the bargaining protocol. The theory extends naturally to games with imperfect public monitoring and heterogeneous discount factors, and yields new insights into classic relational contracting questions.
\end{abstract}

KEYWORDS: Relational contracts, repeated games, self-enforcement, equilibrium selection, bargaining, renegotiation, disagreement.

\section{INTRODUCTION}

MANY ECONOMIC RELATIONSHIPS (such as partnership, employment, and buyer-supplier relationships) are ongoing and governed in whole or in part by self-enforcement. The relational contracts literature studies these relationships using the framework of infinitely repeated games, but repeated games suffer from a vast multitude of equilibria, particularly when players are patient. So equilibrium theory alone offers little hope for predicting behavior or for identifying parameters from observed behavior.

To understand these ongoing relationships, one needs a theory of equilibrium selection. Informally, it seems that players in an ongoing relationship must coordinate closely so as to select "their" equilibrium from the multitude,

\footnotetext{
${ }^{1}$ We thank Nageeb Ali, Jim Andreoni, Sylvain Chassang, Vince Crawford, Marina Halac, Matt Jackson, Sebastian Kranz, Ola Kvaløy, Jin Li, Garey Ramey, Larry Samuelson, Joel Sobel, and several anonymous referees for valuable comments and suggestions, along with seminar participants at Arizona, Collegio Carlo Alberto, Columbia, Duke-Fuqua, ETH Zurich, Florida International, IFPR, Johns Hopkins, Microsoft Research, Penn, Santa Fe Institute, Toronto, UCLA, UCSD, UC Davis, USC, USC-Marshall, Washington University, Western Ontario, and Yale, and conference participants at the GTS Third World Congress, SWET 2008, the 2008 Stony Brook Workshops, the 2009 NBER Organizational Economics Working Group, and NAWMES 2011. Jacob Johnson, Jong-Myun Moon, and Aniela Pietrasz provided excellent research assistance. Miller thanks the NSF (SES-1127643), Microsoft Research, Yale, and the Cowles Foundation for hospitality and financial support; Partha Dasgupta for inspiration to pursue this topic; and his former colleagues at UCSD for all their support. Watson thanks the NSF (SES-0095207 and SES-1227527), the NOAA Fisheries Service, Yale, the Cowles Foundation, and the Center for Advanced Study in the Behavioral Sciences at Stanford for hospitality and financial support.
} 
so it often makes sense to suppose that they coordinate on a Pareto-optimal equilibrium. But this intuition raises two issues. First, payoffs on the Pareto frontier may be supported by the threat of punishments that depart from the Pareto frontier. What if the players can re-coordinate their continuation play to escape from a Pareto-dominated punishment? Second, there are typically many equilibria on the Pareto frontier, where one player's gain is necessarily a loss for others. How do they decide which Pareto-optimal equilibrium to select? Though these issues have arisen in the relational contracts literature, a unified solution is lacking. ${ }^{2}$

We resolve these issues by modeling equilibrium selection as a noncooperative bargaining process embedded in a repeated game with transfers. In each period, the players engage in cheap-talk bargaining via a generalized alternating-offer protocol and make voluntary transfers prior to playing the stage game. We propose three axioms that endow cheap-talk messages with endogenous meaning. Any equilibrium that satisfies these axioms-which we call a contractual equilibrium - has a simple representation. In a contractual equilibrium, the bargaining protocol influences both the welfare level (the sum of the players' payoffs) and the distribution of welfare. These implications have a simple interpretation in terms of relative bargaining power.

We provide an explicit algorithm for constructing contractual equilibria, and we characterize their efficiency and allocative properties. We also extend the theory to games with more than two players, imperfect public monitoring, and heterogeneous discount factors. To demonstrate the utility of contractual equilibrium for the study of relational contracts, we show how the theory generates new insights in two principal-agent applications. We discuss how our theory relates to findings in the empirical macro labor literature and the experimental literature on preplay communication, bargaining, and repeated games. In particular, our results suggest that it may be fruitful for both literatures to explore the role of bargaining power in more depth.

\section{Axioms on Endogenous Meaning}

Section 3 describes our axioms and Section 4 gives them a dynamic programming representation. The first axiom, Internal Agreement Consistency (IAC), ensures that a class of "agreements" has meaning. Specifically, if a player proposes playing as if to switch to a different history in the same equilibrium and if this offer is accepted, then the players play as agreed. By itself, IAC does not change the set of equilibrium payoffs in the game (Theorem 1). A deviant agreement to switch histories can always be discouraged by punishing the player who proposes it and rewarding the one who rejects it.

\footnotetext{
${ }^{2}$ There is also the renegotiation-proofness literature, which addresses the first issue, but generally not the second. We discuss renegotiation proofness later in this section and in more depth in Section 8.
} 
The second axiom, No-Fault Disagreement (NFD), embodies a theory of disagreement. It specifies that if the players do not reach agreement in a given period, then their continuation play should not depend on how bargaining broke down. The idea is that no player should be selectively punished for putting an innovative offer on the table or for rejecting the equilibrium offer in hopes of being able to make an innovative counteroffer. Disagreement also implies zero monetary transfers in the current period, which is equivalent to saying that failure to make an agreed-upon transfer induces disagreement. NFD allows continuation play under disagreement to be Pareto dominated by continuation play under agreement, and it may vary with the past history of actions. By itself, NFD has little influence over the set of equilibrium payoffs in the game (Theorem 2).

Putting IAC and NFD together, however, leads to a significant refinement with a simple representation (Theorem 3(i)). For any given history, the bargaining phase has a well-defined disagreement value and, in equilibrium, the players divide the surplus according to the allocation of bargaining power that arises from the bargaining protocol. Across periods, agreement and disagreement values are linked by enforceability conditions along the lines of Abreu, Pearce, and Stacchetti (1990). The set of equilibrium payoffs available under IAC and NFD is typically much smaller than the set of all subgame-perfect equilibrium payoffs. For instance, in some prisoners' dilemma games, the only possible outcome is infinite repetition of the stage-game equilibrium, regardless of how patient the players are (see Section 5.4).

Generally, there may be multiple, Pareto-ranked payoff sets available under IAC and NFD. The third axiom, Pareto External Agreement Consistency (PEAC), formalizes the intuition that players should be expected to select a Pareto-optimal equilibrium. It ensures meaning for any agreement to play as if switching to an equilibrium that both fully Pareto dominates the current equilibrium and also satisfies IAC and NFD. Under IAC, NFD, and PEAC, the players agree to play a fully Pareto-dominant subgame-perfect equilibrium among those that satisfy IAC and NFD (Theorem 3(ii)).

\section{Characterization of Contractual Equilibrium}

In Section 5, we characterize the set of contractual equilibrium values. Theorem 4 shows that, for any discount factor, this set is a compact line segment of slope -1 . Theorem 5 provides an explicit algorithm for constructing it. The algorithm involves computing two optimal disagreement points that pin down the endpoints, along with an optimal agreement outcome that determines the players' welfare. Theorem 6 provides necessary and sufficient conditions for patient players to attain efficiency. We show that the allocation of bargaining power affects welfare and that welfare is maximized by assigning all bargaining power to one of the players (Theorem 7). Section 6 generalizes contractual equilibrium to games with more than two players, imperfect public monitoring, 
and heterogeneous discount factors. Theorem 8 shows that the set of contractual equilibrium values forms a nonempty, compact hyperpolygon.

\section{Foundations for Relational Contracts}

The relational contracts literature examines how agreements can be selfenforced in environments with repeated play and limited external enforcement. Existing approaches have produced many insights, but mostly skirt around the question of how agents initiate and manage their relationships. ${ }^{3}$ These assumptions simplify analysis, but leave open the implications of bargaining power and how self-enforced agreements evolve after deviations.

Section 7.1 illustrates our approach by applying it to the principal-agent model studied by Levin (2003). Levin applied "strong optimality," a variant of renegotiation proofness that, in this model, implies that the principal cannot make a meaningful deviant offer to the agent, even if she has a monopoly over making proposals. In contrast, in our approach, bargaining power matters: the agent's effort is increasing in his own bargaining power. Surprisingly, the principal's ideal level of bargaining power is intermediate: if she has all the bargaining power, she cannot commit to payments that would motivate the agent; if she has no bargaining power, then the agent extracts all the surplus.

In Section 7.2, we apply contractual equilibrium to the interaction of "explicit" (externally enforced) contracts and "implicit" (self-enforced) contracts, inspired by Baker, Gibbons, and Murphy (1994) and related work by Schmidt and Schnitzer (1995). These papers assume that parties are limited to external enforcement following any deviation, implying that an improvement in external enforcement can reduce equilibrium welfare. In contractual equilibrium, the parties can reevaluate their entire relationship whenever they bargain, so equilibrium welfare always weakly increases in the strength of external enforcement. In Section 7.3, we discuss how the framework developed here can help extend macro labor models to combine moral hazard, bargaining power, and

\footnotetext{
${ }^{3}$ Most approaches simply select an equilibrium on the Pareto frontier (e.g., Radner (1985), Ramey and Watson (1997, 2001), Pearce and Stacchetti (1998), Levin (2002, 2003), Doornik (2006), Fuchs (2007), Rayo (2007), Chassang (2010)). Some assume that after a deviation, the players renegotiate permanently to the optimal spot contract (e.g., Thomas and Worrall (1988), Baker, Gibbons, and Murphy (1994, 2002), Schmidt and Schnitzer (1995), Kvaløy and Olsen (2006, 2009), Hermalin, Li, and Naughton (2013)); Baker, Gibbons, and Murphy (2002) also allowed reallocation of ownership rights. Others allowed for renegotiation once the relationship is underway, but assume that disagreement causes the parties to separate or switch to a stagegame equilibrium permanently (MacLeod and Malcomson (1998), Jackson and Palfrey (1998), Ramey and Watson (2002)). A few employed renegotiation proofness (MacLeod and Malcomson (1989), Levin (2003)). Even those who allowed for continued interaction following disagreement assumed that the players temporarily either receive an exogenous outside option (MacLeod and Malcomson (1989), Levin (2002), Halac (2012), Fong and Li (2010, 2012)) or play an exogenously selected stage Nash equilibrium (Raff and Schmidt (2000), Klimenko, Ramey, and Watson (2008)).
} 
continued interaction under disagreement. We also discuss connections with the empirical literature, where there are suggestive results that link differences in relative bargaining power to productive outcomes.

\section{Contrast With Renegotiation Proofness}

The literature on renegotiation proofness in infinitely repeated games, initiated by Rubinstein (1980), Bernheim and Ray (1989), Farrell and Maskin (1989), and Pearce (1987), addresses the problem of Pareto-dominated continuation play by ruling it out. That is, for a given equilibrium, if the continuation from any particular history is dominated by a qualified alternative, then the equilibrium is removed from consideration. So unlike contractual equilibrium, renegotiation proofness does not model renegotiation explicitly, so it does not contemplate the possibility of disagreement. By capturing the effect of bargaining power in a tractable way, contractual equilibrium yields substantively different testable implications about the players' welfare than does renegotiation proofness. Section 5.4 illustrates the comparison between these approaches and Section 8 discusses their relationship in more depth.

\section{REPEATED GAMES WITH BARGAINING}

\subsection{Extensive Form}

Consider a repeated game augmented with cheap-talk bargaining and transferable utility. Formally, a two-player game in this class is defined by a stage game $\langle\mathcal{A}, u\rangle$, a common discount factor $\delta \in(0,1)$, and a bargaining protocol that we describe shortly. Here $\mathcal{A} \equiv \mathcal{A}_{1} \times \mathcal{A}_{2}$ is the space of action profiles and $u: \mathcal{A} \rightarrow \mathbb{R}^{2}$ is the stage-game payoff function. We normalize the payoffs by $1-\delta$ as is standard. Each period comprises four phases: (i) the public randomization phase, (ii) the bargaining phase, (iii) the transfer phase, and (iv) the action phase. In the public randomization phase, the players observe an arbitrary public randomization device. ${ }^{4}$ In the transfer phase, the players simultaneously make voluntary, nonnegative monetary transfers; that is, each player decides how much money to pay to the other, where money enters their utility quasilinearly. In the action phase, the players play the stage game $\langle\mathcal{A}, u\rangle$. The stage game can also include voluntary transfers that occur simultaneously with other actions. There is no external enforcement of any kind.

In the bargaining phase, the players engage in a generalized alternating-offer bargaining protocol. The number of potential rounds of bargaining may be finite or infinite, but all these rounds occur in a mere instant, so bargaining does not delay the later phases of the period. (If bargaining involved delay, it

\footnotetext{
${ }^{4}$ Such devices are standard in repeated games analysis (see Fudenberg and Maskin (1986)). Here, the device should be viewed as having been constructed by the players in their process of equilibrium selection.
} 
would not be cheap talk.) There is an exogenous random recognition process $\rho \in(\Delta\{1,2\})^{\infty}$ that selects one of the players to make a verbal statement in each round of bargaining, where $\rho_{i, \ell}$ is the probability that player $i$ is recognized in round $\ell$. The selected player-called the proposer-selects a proposal from some language set $\mathcal{L}$ and this is observed by the other player, who is called the responder. If the response is "yes," indicating the responder's acceptance, then the bargaining phase ends and the game proceeds to the transfer phase. If the response is "no," then bargaining may either break down or continue to another round. If bargaining breaks down, then play proceeds to the transfer phase. Breakdown is triggered randomly by a process $\beta \in[0,1]^{\infty}$ with $\prod_{\ell=1}^{\infty}\left(1-\beta_{\ell}\right)=0$, where $\beta_{\ell}$ is the probability that breakdown occurs after a "no" response in bargaining round $\ell$. Both the recognition process and the breakdown process are invariant to the time period and the history; they depend only on the round of bargaining within a period.

The language $\mathcal{L}$ should be sufficiently large that each player can use it to propose to the other how to coordinate their continuation play. ${ }^{5}$ Specifically, we assume that $\mathcal{L}$ contains the space of possible continuation payoff vectors from the voluntary transfer phase, so that a proposal $w \in \mathbb{R}^{2}$ should be interpreted as a suggestion to coordinate on play in the continuation game to achieve $w$ as the continuation value.

\section{AssumPTION 1-Rich Language: It is the case that $\mathbb{R}^{2} \subset \mathcal{L}$.}

Next we develop some notation for histories. We start by describing outcomes of the bargaining phase within a period. Suppose bargaining lasts $\ell$ rounds, at which point an offer is accepted or there is exogenous breakdown. Such an outcome is fully described by an element of $(\{1,2\} \times \mathcal{L})^{\ell} \times$ \{agreement, breakdown\}, where, for each round of bargaining, $\{1,2\}$ accounts for the identity of the proposer and $\mathcal{L}$ accounts for the proposal. For the first $\ell-1$ rounds, the response was "no." Then, for round $\ell$, "agreement" signifies that the last proposal was accepted, whereas "breakdown" signifies that bargaining broke down. Thus the set of possible bargaining outcomes is $\mathcal{B} \equiv \bigcup_{\ell=1}^{\infty}(\{1,2\} \times \mathcal{L})^{\ell} \times$ agreement, breakdown $\}$.

The set of full-period histories, including the "null history," is $\mathcal{H} \equiv \bigcup_{t=0}^{\infty}(\Omega \times$ $\left.\mathcal{B} \times \mathbb{R}_{+} \times \mathbb{R}_{+} \times \mathcal{A}\right)^{t}$, where $\Omega$ is the state space of the arbitrary public randomization device. Here the first $\mathbb{R}_{+}$accounts for transfers from player 1 to player 2 , and the second $\mathbb{R}_{+}$accounts for transfers from player 2 to player 1 . The net of these transfers is summarized by a transfer vector in $\mathbb{R}_{0}^{2} \equiv\left\{m \in \mathbb{R}^{2} \mid m_{2}=-m_{1}\right\}$.

\footnotetext{
${ }^{5}$ One might wish to assume that $\mathcal{L}$ contains descriptions of all continuation strategy profiles in the game. However, since such a construction would be circular (strategies would specify historydependent statements in each negotiation phase, and these statements would include the description of an entire strategy profile), it would lead to a difficult technical issue regarding whether an appropriate "universal language" exists.
} 
A history to the transfer phase is an element of $\mathcal{H} \times \Omega \times \mathcal{B}$. A history to the action phase is an element of $\mathcal{H} \times \Omega \times \mathcal{B} \times \mathbb{R}_{+} \times \mathbb{R}_{+}$. A history to the transfer phase or to the action phase is under agreement if the just-completed bargaining phase ended with "agreement"; otherwise the history is under disagreement.

\subsection{Recursive Characterization of Equilibrium Values}

We characterize the set of subgame-perfect equilibrium (SPE) payoffs by extending the recursive methods of Abreu, Pearce, and Stacchetti (1990), working backward through the phases of a given period. Consider first the action phase. Let $\hat{\Delta} \mathcal{A}$ be the set of probability distributions over $\mathcal{A}$ that are independent across dimensions, that is, the set of mixed action profiles that can arise from independent mixed actions for each player. In equilibrium, the mixed action profile must constitute a Nash equilibrium, taking the players' continuation play from the following period as given. Formally, if $g: \mathcal{A} \rightarrow \mathbb{R}^{2}$ gives the continuation value as a function of the realized action profile $a \in \mathcal{A}$ and if $\alpha \in \hat{\Delta} \mathcal{A}$ is a Nash equilibrium of $\langle\mathcal{A},(1-\delta) u+\delta g\rangle$, then $g$ enforces $\alpha$. In this case, the continuation value from the action phase is

$$
w=(1-\delta) u(\alpha)+\delta g(\alpha)
$$

If $W \subset \mathbb{R}^{2}$ is the set of feasible continuation values starting next period, then the set of continuation values supported from the action phase of the current period is

$$
\begin{aligned}
D(W) \equiv & \left\{w \in \mathbb{R}^{2} \mid\right. \\
& \exists g: \mathcal{A} \rightarrow W \text { and } \alpha \in \hat{\Delta} \mathcal{A} \\
& \text { s.t. } g \text { enforces } \alpha \text { and Eq. (1) holds }\} .
\end{aligned}
$$

Define $\bar{D}_{i}(W) \equiv \bigcup_{w \in D(W)}\left[w_{i}, \infty\right)$ and let $\bar{D}(W) \equiv \bar{D}_{1}(W) \times \bar{D}_{2}(W)$. The set of continuation values supported from the transfer phase is

$$
\begin{aligned}
C(W) \equiv & \left\{w \in \mathbb{R}^{2} \mid\right. \\
& \exists m \in \mathbb{R}_{0}^{2} \text { and } w^{\prime} \in D(W) \\
& \text { s.t. } \left.w=(1-\delta) m+w^{\prime} \text { and } w \in \bar{D}(W)\right\} .
\end{aligned}
$$

To understand how values in $C(W)$ can be achieved, consider any $w, m$, and $w^{\prime}$ that satisfy the conditions of Eq. (3). Without loss of generality, take the case of $m_{1} \geq 0$ so that player 2 is supposed to make a transfer to player 1 . Prescribe that player 2 make this transfer and then the players continue with behavior to achieve $w^{\prime}$ from the action phase. If player 2 does not make the required transfer, then the players coordinate on behavior to achieve a value 
$\underline{w} \in D(W)$ from the action phase, where $\underline{w}$ is selected so that $w_{2} \geq \underline{w}_{2}$. Clearly the prescribed behavior is rational from the transfer phase. ${ }^{6}$

Operator $C$ provides the basis for the recursive formulation of SPE payoffs. Given a set of equilibria $S$, let $V(S)$ be the set of continuation values attained by $S$ starting from any full-period history. When $V$ is evaluated at a singleton set $\{s\}$, we call it the value set of $s$ and abuse notation to write $V(s)$. Let $S_{\mathrm{SPE}}$ be the set of all subgame-perfect equilibria of our model with bargaining. From the construction above, it is clear that $V\left(S_{\mathrm{SPE}}\right)$ is the largest fixed point of $\operatorname{co} C(\cdot)$, where co denotes the convex hull to account for public randomization at the beginning of each period. Also observe that $V\left(S_{\mathrm{SPE}}\right)$ is identical to the set of subgame-perfect equilibrium payoffs in an otherwise identical game with no bargaining phase, denoted $V\left(S_{\mathrm{NB}}\right){ }^{7}$

\section{AXIOMATIZATION OF ENDOGENOUS MEANING}

So far, statements in $\mathcal{L}$ neither have any particular meaning nor impose any constraints on the set of equilibrium payoffs. We next develop axioms that refine the equilibrium set by imposing meaning in equilibrium. Our axioms are in the spirit of equilibrium restrictions introduced by the literature on preplay communication, as discussed in Section 8.

\subsection{A Foundation for Meaningful Proposals}

In an equilibrium $s \in S_{\mathrm{SPE}}$, the players recognize that any payoff in $C(\operatorname{co} V(s))$ is attainable from the transfer phase by randomizing over continuation values achieved by $s$ using the public randomization device at the beginning of the next period. We posit that the players should have the opportunity to agree on such a payoff and then follow through on their agreement; that is, any proposal to play as if conditionally switching to different histories in the same equilibrium should be taken seriously. Our first axiom requires that, on or off the equilibrium path, if there is agreement on a continuation value $w$ that is consistent with the equilibrium strategy profile (the proposer offers $w \in C(\operatorname{co} V(s))$ and the responder says "yes"), then the players proceed in a way that achieves value $w$.

\footnotetext{
${ }^{6}$ Nothing more can be supported since feasibility requires $w=(1-\delta) m+w^{\prime}$ for some $m \in \mathbb{R}_{0}^{2}$ and $w^{\prime} \in D(W)$, and each player $i$ can guarantee himself some value in $\bar{D}_{i}(W)$ by making a zero transfer. Also note that it is not helpful to have both players make positive transfers-one could reduce both transfers equally until one equals zero, loosening the incentive constraints without affecting the continuation value. For more details, see Goldlücke and Kranz (2012).

${ }^{7}$ The players can always ignore the bargaining phase, so $V\left(S_{\mathrm{SPE}}\right) \supseteq V\left(S_{\mathrm{NB}}\right)$. Similarly, since both games feature arbitrary public correlation devices and the bargaining phase is pure cheap talk, $V\left(S_{\mathrm{SPE}}\right) \subseteq V\left(S_{\mathrm{NB}}\right)$.
} 
AXIOM IAC-Internal Agreement Consistency: For every history to the transfer phase under agreement, if the agreement satisfies $w \in C(\operatorname{co} V(s))$, then continuation play yields value $w^{8}$

Let $S_{\mathrm{IAC}}$ be the subset of $S_{\mathrm{SPE}}$ that satisfies IAC. Note that IAC does not require the players to reach agreement. It merely defines the meaning of a class of agreements, refining the equilibrium set due to the consequences in off-equilibrium-path contingencies. Though IAC eliminates some equilibria, it does not on its own refine the equilibrium value set.

\section{THEOREM 1-IAC Has No Bite: It is the case that $V\left(S_{\mathrm{IAC}}\right)=V\left(S_{\mathrm{SPE}}\right)$.}

The proof, in Appendix A.1, actually strengthens the result by invoking a stronger consistency axiom that also addresses agreements to switch to continuations that are supported by other equilibria. Under IAC there may be deviant proposals that, if implemented, would make the proposer better off than if he made his equilibrium proposal. However, it is always possible to deter a deviant proposal by specifying that if the responder says "no," then the players should coordinate to punish the proposer and reward the responder. In this way, the agreement consistency axiom is undermined by the fact that continuation play can vary in arbitrary ways following a disagreement. To narrow the set of equilibrium values, we need a theory of disagreement.

\subsection{A No-Fault Theory of Disagreement}

We next add a no-fault disagreement axiom that embodies a theory of how players behave under disagreement. It requires that continuation play in the absence of agreement - though it may vary with the history of actions, transfers, and past agreements-should not vary with the manner in which bargaining broke down.

Disagreement can arise in myriad ways. Bargaining may randomly break down after a responder says "no," following any sequence of equilibrium or deviant offers. That disagreement play should not be sensitive to the manner of breakdown represents the nostrum that "nothing is agreed until everything is agreed." That is, no player can be singled out for punishment for making an innovative, deviant offer that is rejected or for rejecting an offer in hopes of being able to make an innovative counteroffer. We also assume that play from the action phase under disagreement is insensitive to the transfers just made so

\footnotetext{
${ }^{8}$ For simplicity, we state IAC in a somewhat stronger form than is necessary. The necessary part is that the players should achieve the value of an agreement $w$ if it is attainable with a transfer and any continuation $w^{\prime} \in D(\operatorname{co} V(s))$ that is actually attained by $s$ after some history to the action phase.
} 
as to capture the idea that a player can void an agreement by refusing to make the required up-front payment. ${ }^{9}$

AXIOM NFD—No-Fault Disagreement: For every history to the action phase under disagreement, continuation play is measurable with respect to the full-period history at the end of the previous period and the current-period realization of the public randomization device. ${ }^{10}$

Although NFD constrains the meaning of disagreements, by itself NFD does little to constrain the set of attainable payoffs. In particular, in the realm of pure-strategy equilibria, NFD does not alter the Pareto boundary of equilibrium continuation values; neither does it eliminate any payoffs attainable in a subgame-perfect equilibrium without transfers.

To state this result, we need to introduce some extra notation; the proof is given in Appendix A.1. Let $S_{\mathrm{NFD}}$ be the subset of $S_{\mathrm{SPE}}$ that satisfies NFD. Let $S_{\mathrm{SPE}}^{\mathrm{ps}}$ be the set of pure-strategy subgame-perfect equilibria, let $S_{\mathrm{NFD}}^{\mathrm{ps}}$ be the subset of pure-strategy equilibria that satisfy NFD, and let $S_{\mathrm{SPE}}^{\text {nt }}$ be the set of subgame-perfect equilibria that specify zero transfers after all histories. For any set of payoffs $W \subset \mathbb{R}^{2}$, let $P(W)$ be the Pareto frontier of $W$.

THEOREM 2-NFD Has Little Bite: It is the case that $P\left(V\left(S_{\mathrm{SPE}}^{\mathrm{ps}}\right)\right)=$ $P\left(V\left(S_{\mathrm{NFD}}^{\mathrm{ps}}\right)\right)$ and $V\left(S_{\mathrm{SPE}}^{\mathrm{nt}}\right) \subseteq V\left(S_{\mathrm{NFD}}\right)$.

In combination with IAC, however, NFD has strong effects. In particular, it rules out the kinds of punishments used to discourage deviant proposals in the proof of Theorem 1. Following any history, the bargaining phase (viewed in isolation as a game in itself) has a unique disagreement outcome by NFD, and a nondegenerate bargaining set by IAC. Therefore, it has unique subgameperfect equilibrium, as established by the literature on alternating-offer bargaining (Binmore (1987), Rubinstein (1982), Shaked and Sutton (1984)). We characterize the implications of NFD and IAC in Section 4.

Although the combination of NFD and IAC eliminates many equilibria, multiple Pareto-ranked equilibrium payoffs generally still survive. Our third axiom allows the players to meaningfully discuss whether to switch to an equilibrium that is "better" than the one they are currently playing. The following definition formalizes what makes one equilibrium better than another.

\footnotetext{
${ }^{9}$ In other words, transfers "seal the deal." In the realm of relational contracts, where there is always at least a rudimentary legal system in the background, this interpretation embodies the fact that signing a contract can create a legal obligation to make an unconditioned payment, even when no other contract clauses are legally enforceable. Adopting this interpretation formally would require extra notation to keep track of the transfer required in agreement. Then, with a slightly different version of IAC, we would obtain the same implications.

${ }^{10}$ Again, for simplicity, we state the axiom in a simpler, stronger form than needed. Section 8.1 discusses how NFD could be weakened without affecting the results.
} 
DEFINITION 1: A payoff set $W^{\prime} \subset \mathbb{R}^{2}$ fully Pareto dominates another payoff set $W$ if (i) for every $v^{\prime} \in W^{\prime}$, there is no $v \in W \backslash W^{\prime}$ that satisfies $v \geq v^{\prime}$ (componentwise), and (ii) for every $v \in W$, there exists $v^{\prime} \in W^{\prime}$ such that $v^{\prime} \geq v$ (componentwise).

We say that a strategy profile $s^{\prime}$ fully Pareto dominates another strategy profile $s$ if $\operatorname{co} V\left(s^{\prime}\right)$ fully Pareto dominates $\operatorname{co} V(s)$. Because full Pareto dominance is a demanding notion of dominance, the following axiom - that the players must honor any agreement to deviate to an equilibrium that fully Pareto dominates their current equilibrium-is relatively weak.

AXIOM PEAC-Pareto External Agreement Consistency: For every history to the action phase under agreement in equilibrium $s$, if the agreement satisfies $w \in$ $C\left(\operatorname{co} V\left(s^{\prime}\right)\right)$, where $s^{\prime} \in S_{\mathrm{IAC}} \cap S_{\mathrm{NFD}}$ and $\operatorname{co} V\left(s^{\prime}\right)$ fully Pareto dominates $\operatorname{co} V(s)$, then continuation play yields value $w$.

As with IAC, Appendix A.1 shows that PEAC has no bite.

COROLLARY 1-PEAC Has No Bite: We have $V\left(S_{\mathrm{PEAC}}\right)=V\left(S_{\mathrm{SPE}}\right)$.

But the three axioms together yield a sharp characterization. We define a contractual equilibrium to be any subgame-perfect equilibrium that satisfies IAC, NFD, and PEAC. Accordingly, let $S_{\text {PEAC }}$ be the set of equilibria that satisfy PEAC and let $S_{\mathrm{CE}} \equiv S_{\mathrm{IAC}} \cap S_{\mathrm{NFD}} \cap S_{\mathrm{PEAC}}$. The next section shows that $V\left(S_{\mathrm{CE}}\right)$ is identical to a "dominant bargaining self-generated set" that we identify. The rest of this paper is devoted to characterizing this set.

\section{REPRESENTATION}

To characterize contractual equilibrium values, we compare the bargaining phase in an individual period to a simple bargaining game in which (i) the players bargain directly over a fixed set of payoff vectors and (ii) the generalized alternating-offer protocol is of the form described in Section 2.1. Analysis of this simple bargaining game yields a "bargaining operator" that we embed in a recursive construction of continuation values.

In the simple bargaining game, the players bargain over the selection of a payoff vector from a set $W \subset \mathbb{R}^{2}$. If they fail to reach an agreement, then the payoff vector is some value $\underline{w} \in W$. Suppose that the sum of the players' continuation payoffs - which we call the welfare level —is constant along the Pareto boundary of $W$, and suppose this boundary includes all points above $w$ with the same welfare. Then the unique subgame-perfect equilibrium payoff vector $w$ satisfies

$$
w_{1}+w_{2}=\max _{w^{\prime} \in W}\left(w_{1}^{\prime}+w_{2}^{\prime}\right) \quad \text { and } \quad w=\underline{w}+\pi\left(w_{1}+w_{2}-\underline{w}_{1}-\underline{w}_{2}\right),
$$


where for each player $i, \pi_{i}=\sum_{\ell=1}^{\infty}\left(\rho_{i, \ell} \beta_{\ell} \prod_{k=1}^{\ell-1}\left(1-\beta_{k}\right)\right)$. In other words, the players maximize welfare, and they split the surplus in fractions $\pi_{1}$ and $\pi_{2}$. This conclusion is stated in Lemma 6 in Appendix A.2. Let $\pi=\left(\pi_{1}, \pi_{2}\right)$ and note that $\pi_{1}+\pi_{2}=1$.

Next we construct a set of payoff vectors by calculating the equilibrium payoffs that can arise in the simple bargaining game when $\underline{w}$ can be any disagreement value in $\underline{W}$ :

$$
B(W, \underline{W}) \equiv\left\{\underline{w}+\pi\left(\max _{w \in W}\left(w_{1}+w_{2}\right)-\underline{w}_{1}-\underline{w}_{2}\right) \mid \underline{w} \in \underline{W}\right\} .
$$

Returning to the repeated game, recall that operators $D$ and $C$ produce the sets of continuation values from the action phase and transfer phase of a given period, respectively, as a function of a set of continuation values $W$ from the start of the following period. We interpret $C(W)$ as the set of payoff-vector alternatives for the players in the bargaining phase and interpret $D(W)$ as the set of disagreement payoffs attainable from the transfer phase (with transfers of zero). The bargaining operator, along with public randomization at the beginning of the period, yields the compound operator co $B(C(\cdot), D(\cdot)) .{ }^{11}$ We examine fixed points of this compound operator.

DEFINITION 2: A nonempty set $W \subset \mathbb{R}^{2}$ is called a bargaining self-generated $(B S G)$ set if $W=\operatorname{co} B(C(W), D(W))$.

Our next definition combines bargaining self-generation with full Pareto dominance.

DEFinition 3: A BSG set $W^{*} \subset \mathbb{R}^{2}$ is dominant if it fully Pareto dominates every other BSG set.

By definition, there can be at most one dominant BSG set. Our representation theorem establishes a relation between sets of contractual equilibrium values and bargaining self-generated sets. The theorem shows that the value set of every contractual equilibrium is contained in the dominant BSG set, and for each value in the dominant BSG set, there is a contractual equilibrium that delivers it.

THEOREM 3-Representation: Given a game $\langle\mathcal{A}, u, \delta, \rho, \beta\rangle$, let $\pi_{i}=$ $\sum_{\ell=1}^{\infty}\left(\rho_{i, \ell} \beta_{\ell} \prod_{k=1}^{\ell-1}\left(1-\beta_{k}\right)\right)$ for each $i$. Then the following properties hold:

(i) If $W$ is a $B S G$ set, then $W \subseteq V\left(S_{\mathrm{IAC}} \cap S_{\mathrm{NFD}}\right)$; furthermore, if $s \in S_{\mathrm{IAC}} \cap$ $S_{\mathrm{NFD}}$, then $\operatorname{co} V(s)$ is a $B S G$ set.

(ii) The set $V\left(S_{\mathrm{CE}}\right)$ is the dominant BSG set.

\footnotetext{
${ }^{11}$ Recall that $D(W) \subset C(W)$, welfare is constant along the Pareto boundary of $C(W)$, and $C(W)$ extends to the edges of $D(W)$ along each player's axis. This implies that $B(C(\cdot), D(\cdot))$ is well-defined.
} 
The proof is given in Appendix A.2. In principle, every point in $V\left(S_{\mathrm{CE}}\right)$ is an equally good candidate to be selected as the equilibrium payoff in the first period. By construction, the first period payoff must be $v=B\left(C\left(V\left(S_{\mathrm{CE}}\right)\right),\{\underline{w}\}\right)$, where $\underline{w} \in D\left(V\left(S_{\mathrm{CE}}\right)\right)$ is the payoff that arises if the players disagree in the first period. But nothing pins down which point in $D\left(V\left(S_{\mathrm{CE}}\right)\right)$ is selected to be the disagreement point in the first period-neither the axioms nor any prior agreement. The same is true of the disagreement payoff in any period following a history in which the players have never agreed. So the problem of selecting a particular equilibrium boils down to determining how the players will play if they have never agreed. In applications, this behavior might arise from custom, the status quo, or social institutions.

Note that the bargaining-protocol parameters $\rho$ and $\beta$ enter the definition of the compound operator co $B(C(\cdot), D(\cdot))$ in ways summarized by $\pi_{1}$ and $\pi_{2}$. Thus, the recursive construction of contractual equilibrium values can use a simplified description of the game, given by $\langle\mathcal{A}, u, \delta, \pi\rangle$. Also, the standard connection between the subgame-perfect equilibrium of the noncooperative bargaining game and a cooperative bargaining solution applies here. In particular, the equilibrium payoff $w$ shown in Eq. (4) coincides with the generalized Nash (1950) bargaining solution with weights $\pi_{1}$ and $\pi_{2}$. Thus, we call $\pi$ a vector of "bargaining weights," understanding that these weights represent the bargaining protocol.

\section{CHARACTERIZATION}

This section characterizes the set of contractual equilibrium values $V\left(S_{\mathrm{CE}}\right)$ and provides an algorithm for computing it. This section also provides results on efficiency and how bargaining power affects what values are attainable. The characterization shows that every BSG set (and hence $V\left(S_{\mathrm{CE}}\right)$ ) is a line segment with slope -1 . Equivalently, every BSG set has two endpoints $z^{1}, z^{2} \in \mathbb{R}$, satisfying $z_{1}^{1}+z_{2}^{1}=z_{1}^{2}+z_{2}^{2}$. Let $z^{1}$ be the endpoint that favors player 2 ("punishing" player 1) and let $z^{2}$ be the endpoint that favors player 1 . For any such set $W$, we define $\operatorname{span}(W) \equiv z_{1}^{2}-z_{1}^{1}=z_{2}^{1}-z_{2}^{2}$ and level $(W) \equiv z_{1}^{1}+z_{2}^{1}=z_{1}^{2}+z_{2}^{2}$, which are the vertical distance, or payoff span, across $W$ and the joint value, or welfare level, respectively, of each point in $W$.

\subsection{Existence and Construction}

We begin by showing that $V\left(S_{\mathrm{CE}}\right)$ exists for any discount factor.

THEOREM 4-Existence: For a game $\langle\mathcal{A}, u, \delta, \pi\rangle$, if $\mathcal{A}$ is finite, then $V\left(S_{\mathrm{CE}}\right)$ is a nonempty, compact line segment of slope -1 .

The next result provides a complete, constructive characterization of $V\left(S_{\mathrm{CE}}\right)$. 
THEOREM 5-Construction: For a game $\langle\mathcal{A}, u, \delta, \pi\rangle$, if $\mathcal{A}$ is finite and $W^{*}=$ $V\left(S_{\mathrm{CE}}\right)$, then the following properties hold:

(i) The $\operatorname{span}\left(W^{*}\right)$ is equal to the maximal fixed point of $\Gamma \equiv \gamma^{2}+\gamma^{1}$, where for players $i \neq j$,

$$
\begin{gathered}
\gamma^{j}(d) \equiv \max _{\eta, \alpha}\left(\pi_{j} u_{i}(\alpha)-\pi_{i} u_{j}(\alpha)+\frac{\delta}{1-\delta} \eta(\alpha)\right) \\
\text { s.t. } \quad\left\{\begin{array}{c}
\eta: \mathcal{A} \rightarrow[-d, 0], \text { extended to } \hat{\Delta} \mathcal{A} \\
\alpha \in \hat{\Delta} \mathcal{A} \text { is a Nash equilibrium } \\
o f\left\langle\mathcal{A}_{i} \times \mathcal{A}_{j},(1-\delta)\left(u_{i}, u_{j}\right)+\delta(\eta,-\eta)\right\rangle .
\end{array}\right.
\end{gathered}
$$

(ii) level $\left(W^{*}\right)$ is equal to $\max _{\eta, \alpha}\left(u_{1}(\alpha)+u_{2}(\alpha)\right)$

$$
\text { s.t. }\left\{\begin{array}{l}
\eta: \mathcal{A} \rightarrow\left[0, \operatorname{span}\left(W^{*}\right)\right], \text { extended to } \hat{\Delta} \mathcal{A} \\
\alpha \in \hat{\Delta} \mathcal{A} \text { is a Nash equilibrium of }\langle\mathcal{A},(1-\delta) u+\delta(\eta,-\eta)\rangle .
\end{array}\right.
$$

(iii) The endpoints of $W^{*}$ are

$$
\begin{aligned}
& z^{1}=(-1,1) \gamma^{1}\left(\operatorname{span}\left(W^{*}\right)\right)+\pi \operatorname{level}\left(W^{*}\right), \\
& z^{2}=(1,-1) \gamma^{2}\left(\operatorname{span}\left(W^{*}\right)\right)+\pi \operatorname{level}\left(W^{*}\right) .
\end{aligned}
$$

The rest of this subsection contains the proof of Theorems 4 and 5. We proceed with a series of lemmas that characterize the geometry of BSG sets and the relation between them; we then identify a dominant BSG set. Proofs of the first three lemmas are presented in Appendix A.3.

LEMMA 1: If $W \subset \mathbb{R}^{2}$ is a BSG set, then it is a bounded, convex subset of a line with slope -1 .

Thus all points in $W$ have the same welfare level. Observe that it is possible for a BSG set to not contain one or both of its endpoints. We first focus attention on BSG sets that are closed; later we argue that any open BSG set is fully Pareto dominated by $V\left(S_{\mathrm{CE}}\right)$, which is closed. So consider the endpoints $z^{1}$ and $z^{2}$ of an arbitrary closed BSG set. As $z^{1}$ and $z^{2}$ are extreme points of $B(W, \underline{W})$, there exist disagreement points $\underline{w}^{1}, \underline{w}^{2} \in \underline{W}$ relative to which $z^{1}$ and $z^{2}$ are the bargaining outcomes that satisfy Eq. (4). We will express $z^{1}$ and $z^{2}$ in relation to optimization problems parameterized by the welfare level and payoff span of $W$.

LEMMA 2: For a given closed BSG set $W, z_{1}^{2}=\pi_{1} \operatorname{level}(W)+\gamma^{2}(\operatorname{span}(W))$ and $z_{2}^{1}=\pi_{2}$ level $(W)+\gamma^{1}(\operatorname{span}(W))$. Furthermore, $\gamma^{1}(d)$ and $\gamma^{2}(d)$ exist and satisfy $\gamma^{2}(d)+\gamma^{1}(d) \geq 0$ for all $d \geq 0$. 
The proof operates by solving the problem of maximizing a player's payoff that can be supported by continuation values in $W$ as a function of the welfare level and payoff span of $W$. Now we can compare BSG sets by using the functions $\gamma^{1}$ and $\gamma^{2}$. We find that BSG sets are ranked by full Pareto dominance, since a larger set of continuation values-regardless of its position in $\mathbb{R}^{2}$-supports a larger range of stage-game action profiles.

LEMma 3: Suppose that $W$ is a closed BSG set and $W^{\prime}$ is any BSG set. If $\operatorname{span}(W) \geq \operatorname{span}\left(W^{\prime}\right)$, then $W$ fully Pareto dominates $W^{\prime}$.

We next use the functions $\gamma^{1}$ and $\gamma^{2}$ to prove the existence of a fully Paretodominant BSG set. First, for any BSG set $W$ with endpoints $z^{1}$ and $z^{2}$,

$$
\operatorname{span}(W)=z_{1}^{2}-z_{1}^{1}=z_{1}^{2}-\left(\operatorname{level}(W)-z_{2}^{1}\right)=z_{1}^{2}+z_{2}^{1}-\operatorname{level}(W) .
$$

Substituting for $z_{1}^{2}$ and $z_{2}^{1}$ using Lemma 2 yields $\operatorname{span}(W)=\gamma^{2}(\operatorname{span}(W))+$ $\gamma^{1}(\operatorname{span}(W))$. Thus, the payoff span of a BSG set must be a fixed point of $\Gamma$. Further, as the analysis underlying Lemma 2 makes clear, every fixed point of $\Gamma$ is the payoff span of a BSG set.

Lemma 4: The function $\Gamma$ has a maximal fixed point $d^{*} \geq 0$.

ProOF: Observe that $\Gamma$ is nondecreasing because an increase in payoff span relaxes the constraints in the problems that define $\gamma^{1}$ and $\gamma^{2}$. The function $\Gamma$ is bounded because $u$ is bounded and $\delta$ is fixed. By Tarski's fixed-point theorem, $\Gamma$ has a maximal fixed point, which is nonnegative because $\Gamma(0) \geq 0$. Q.E.D.

To construct $W^{*}$ from $d^{*}$, let $\lambda^{*}$ be the value of $\max _{\eta, \alpha}\left(u_{1}(\alpha)+u_{2}(\alpha)\right)$ subject to the constraints in Eq. (7), using $d^{*}$ in place of $\operatorname{span}(W)$. Then construct the endpoints $z^{1}$ and $z^{2}$ by Eq. (8), using $d^{*}$ in place of $\operatorname{span}(W)$ and $\lambda^{*}$ in place of level $(W)$. By construction, $W^{*} \equiv \operatorname{co}\left\{z^{1}, z^{2}\right\}$ is a BSG set and its span is maximal over all BSG sets. By Lemma $3, W^{*}$ fully Pareto dominates all other closed BSG sets.

To complete the proof, we return to the case of BSG sets that may not be closed. Taking the closure of a BSG set does not necessarily form a BSG set, because new Nash equilibria could emerge in the dynamic program. But for any open BSG set, the following lemma shows that there exists a larger, closed BSG set.

LEMMA 5: If $W$ is a BSG set that is not closed, then there exists a closed BSG set that fully Pareto dominates $W$.

Proof: Let $W$ be an open BSG set. Define $\hat{\gamma}^{j}(d)$ to be the same as $\gamma^{j}(d)$ except that (i) its objective is a supremum rather than a maximum 
and (ii) the range of $\eta$ is defined as the open interval $(-d, 0)$ rather than $[-d, 0]$. Then $\operatorname{span}(W)$ is a fixed point of $\hat{\gamma}^{2}+\hat{\gamma}^{1}$. By construction, $\hat{\gamma}^{2}+\hat{\gamma}^{1} \leq$ $\gamma^{2}+\gamma^{1}$, so $\Gamma(\operatorname{span}(W)) \geq \operatorname{span}(W)$. Because $\Gamma$ is increasing and bounded, $d^{*} \geq \operatorname{span}(W)$. One can easily confirm that Lemma 2 extends to nonclosed BSG sets, implying that $W^{*}$ fully Pareto dominates $W$. Therefore, $W^{*}$ is not open. A parallel argument implies that $W^{*}$ cannot contain just one of its endpoints.

Q.E.D.

Thus $W^{*}=V\left(S_{\mathrm{CE}}\right)$, proving Theorems 4 and 5 .

\subsection{Efficiency}

To indicate the dependence of $\gamma^{i}$ and $\Gamma$ on $\delta$, we write $\gamma_{\delta}^{i}$ and $\Gamma_{\delta}$. Observe that $\Gamma_{\delta}$ is bounded and increasing in $\delta$. In fact, if $\Gamma_{\delta}(d)>0$ for some $d$ and some $\delta$, then $\Gamma_{\delta^{\prime}}(d) \geq d$ for $\delta^{\prime}$ sufficiently large, implying that $d^{*}$ is bounded away from zero for discount factors close enough to 1 . If this is the case, then any action profile can be supported in a single period if the players are sufficiently patient. Moreover, $\Gamma_{\delta}(\infty)$ does not depend on $\delta$. These observations lead to a necessary and sufficient condition for patient players to attain payoffs on the efficient frontier of the payoff set.

THEOREM 6: If $\Gamma(\infty)>0$, then $d^{*}>0$, and $V\left(S_{\mathrm{CE}}\right)$ is a subset of the efficient frontier for $\delta$ sufficiently close to 1 . If $\Gamma(\infty)=0$, then $V\left(S_{\mathrm{CE}}\right)$ attains the same welfare level as the welfare-maximizing Nash equilibrium of the stage game.

Proofs for the remainder of this section are provided in Appendix B.1 of the Supplemental Material (Miller and Watson (2013)), along with methods for constructing efficient contractual equilibria when the best response correspondences satisfy certain properties.

\subsection{The Role of Relative Bargaining Power}

In static bargaining games with transferable utility, the allocation of bargaining power typically has no effect on the level of welfare. In contractual equilibrium, however, the bargaining weights play a critical role in determining the span of $V\left(S_{\mathrm{CE}}\right)$ and, hence, the welfare level attainable in contractual equilibrium. In fact, in general, the highest welfare level is attained when bargaining power is extremely unequal, that is, when one player has a monopoly over the right to propose.

THEOREM 7: Given $\mathcal{A}, \delta$, and $u$, level $\left(V\left(S_{\mathrm{CE}}\right)\right)$ is maximized at either $\pi=$ $(0,1)$ or $\pi=(1,0)$. 
For intuition, consider a BSG set associated with two disagreement points, one of which is closer to the Pareto frontier than the other. The BSG set is the projection of the two disagreement points onto the frontier in the direction of the bargaining shares. Since equal bargaining shares form a vector perpendicular to the frontier, they do not maximize the projected distance between the disagreement points. Even when the stage game is symmetric, extreme bargaining power (in either direction) can yield strictly higher welfare than interior bargaining power. This feature is illustrated by the prisoners' dilemmas in the following subsection. In an asymmetric game, typically one extreme will yield the highest welfare, as displayed in the applications described in Section 7.

\subsection{Application to the Prisoners' Dilemma}

To illustrate contractual equilibrium and compare it with other concepts, consider the class of prisoners' dilemmas

$$
\begin{array}{l|c|c|} 
& \multicolumn{1}{c}{\mathrm{C}} & \multicolumn{1}{c}{\mathrm{D}} \\
\cline { 2 - 3 } \mathrm{C} & 1,1 & -r, x \\
\cline { 2 - 3 } \mathrm{D} & x,-r & 0,0 \\
\cline { 2 - 3 } & &
\end{array}
$$

where $r>0, x>1$, and $x-r<2$. Cooperation can be sustained in a subgameperfect equilibrium if $\delta \geq \frac{x-1}{x}$. In this case, the Pareto frontier of SPE payoff vectors is the entire line segment from $(0,2)$ to $(2,0)$.

We calculate the contractual equilibrium value set $V\left(S_{\mathrm{CE}}\right)$ by applying Theorems 5 and 6 . Observe that if $\frac{\delta}{1-\delta} d \geq \max \{r, 2(x-1)\}$, then

$$
\begin{aligned}
& \gamma^{2}(d)=\max \left\{\pi_{2}-\pi_{1}-(x-1), \pi_{2} x-\pi_{2} r,-\pi_{1} x-\pi_{2} r, 0\right\}, \\
& \gamma^{1}(d)=\max \left\{\pi_{1}-\pi_{2}-(x-1),-\pi_{2} x-\pi_{1} r, \pi_{1} x-\pi_{1} r, 0\right\},
\end{aligned}
$$

where the elements in each maximand are the values at CC, DC, CD, and DD, in this order. Because D is a dominant strategy in the stage game, the values at mixed strategies are convex combinations of these.

Theorem 6 tells us to plug in $d=\infty$ to see whether sufficiently patient players can support $\mathrm{CC}$ along the equilibrium path. It is clear that $\gamma^{2}(\infty)+$ $\gamma^{1}(\infty)>0$ if and only if either $x>r$ or $\left|\pi_{2}-\pi_{1}\right|>x-1$, which by Theorem 6 is necessary and sufficient for $\mathrm{CC}$ to be supported in contractual equilibrium for sufficiently high $\delta<1$. If, on the other hand, $x<r$ and $\left|\pi_{2}-\pi_{1}\right|<x-1$, then $W^{*}=\{(0,0)\}$ regardless of $\delta$. These results also illustrate Theorem 7: for any given prisoners' dilemma, the condition for obtaining efficiency at high discount factors is most relaxed when $\pi_{1}=1$ or $\pi_{2}=1$.

To get a feel for the construction of the set $W^{*}$, consider Figure 1. Suppose that $\pi_{1}=\pi_{2}, x>r$, and $\frac{\delta}{1-\delta}(x-r) \geq r$, so the players attain efficiency. Consider player 1's favorite point $z^{2}$, which is achieved with reference to the 


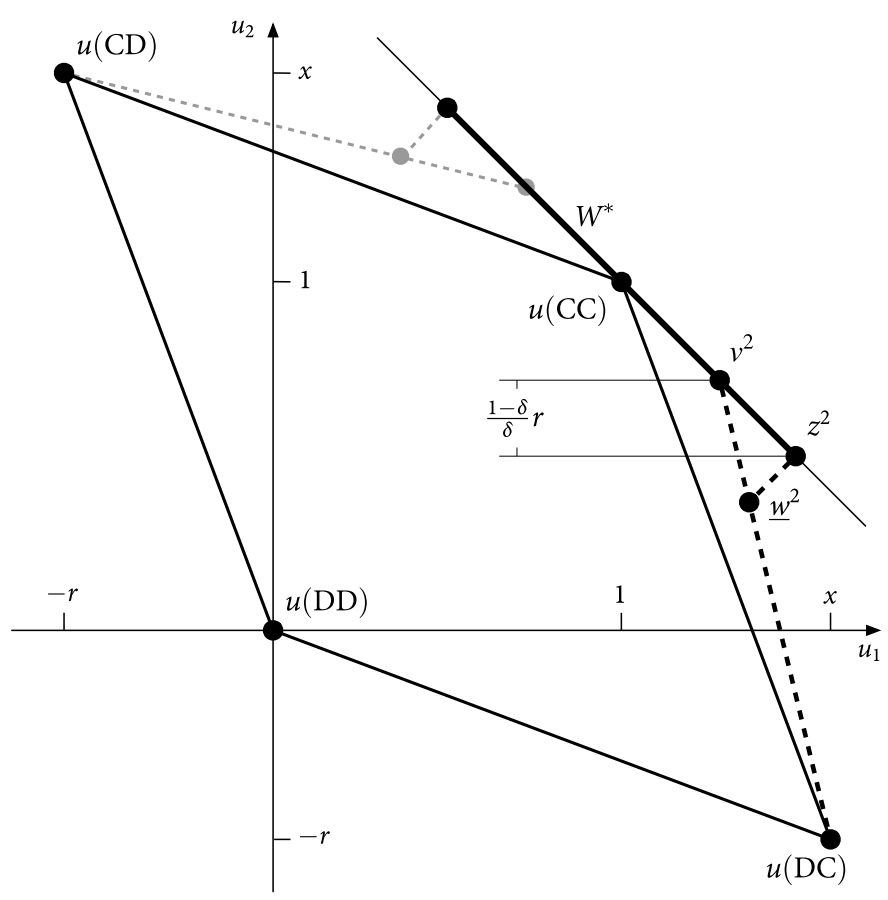

FIGURE 1.-The prisoners' dilemma with equal bargaining power. The parameters satisfy $x>r$ and $\left(\pi_{1}, \pi_{2}\right)=\left(\frac{1}{2}, \frac{1}{2}\right)$. The endpoint $z^{2}$ is attained by playing CC in the stage game and using a transfer to split the surplus relative to the disagreement point $\underline{w}^{2}$. The disagreement point $\underline{w}^{2}$, in turn, is attained by playing DC in the stage game and continuing with promised utility $v^{2}$ if no deviation occurs, but promised utility $z^{2}$ if player 2 deviates. Minimizing $z_{2}^{2}$ subject to enforcing DC under disagreement and CC under agreement requires both $v_{2}^{2}-z_{2}^{2} \geq \frac{1-\delta}{\delta} r$ and $1-z_{2}^{2} \geq \frac{1-\delta}{\delta}(x-1)$.

disagreement point $\underline{w}^{2}$ that is furthest in the direction $\left(\pi_{2},-\pi_{1}\right)=\left(\frac{1}{2},-\frac{1}{2}\right)$. In turn, $\underline{w}^{2}$ is the weighted average of $u(\mathrm{DC})=(x,-r)$ and a point $v^{2} \in W^{*}$. The former gets weight $(1-\delta)$ and represents the payoff in the current period, whereas the latter has weight $\delta$ and gives the continuation payoff from the next period. Player 1 prefers that $v^{2}$ be "pushed" down and to the right. However, $v^{2}$ cannot equal $z^{2}$ because there must be room to punish player 2 if he were to deviate from DC. If player 2 deviates, the continuation value $z^{2}$ is selected; otherwise, the continuation value is $v^{2}$. To discourage player 2's deviation from DC, it must be that $v_{2}^{2}-z_{2}^{2} \geq \frac{1-\delta}{\delta} r$. To support efficiency, it must also be that $1-z_{2}^{2} \geq \frac{1-\delta}{\delta}(x-1)$ to discourage deviations from $\mathrm{CC}$. The construction is pictured in Figure 1.

The contractual equilibrium set is intuitively satisfying in two key ways. First, as Figure 1 demonstrates, contractual equilibrium yields a strict subset of the 
Pareto frontier when $\mathrm{CC}$ is sustained along the equilibrium path. ${ }^{12}$ It can be shown that both endpoints of this subset shift toward higher payoffs for player $i$ when $\pi_{i}$ increases, so (conditional on sustaining CC on the equilibrium path) each player's share of the available welfare is increasing (in a set-ordered sense) in his bargaining power. Second, contractual equilibrium has the appealing property that equilibrium welfare decreases (weakly) as $r$ increases, because higher $r$ makes it more difficult to support play of DC or CD under disagreement.

These properties contrast with the equilibria selected by renegotiationproofness theories. Goldlücke and Kranz (2013; henceforth GK) showed that if renegotiation is applied only before the transfer phase, then "strong optimality" (Levin (2003)), "strong perfection" (Rubinstein (1980)), and "strong renegotiation proofness" (Farrell and Maskin (1989)) all select the entire Pareto frontier of SPE payoffs. For a discount factor of at least $\frac{x-1}{x}$, this is the line segment between $(0,2)$ and $(2,0)$. Contractual equilibrium is more restrictive. For instance, in the case of $\pi_{1}=\pi_{2}=1 / 2$, if $x<r$, then contractual equilibrium selects only the value $(0,0)$, regardless of the discount factor. If $x \geq r$, then the lowest discount factor that supports $\mathrm{CC}$ in a contractual equilibrium is $\max \left\{\frac{r}{x}, \frac{2 x-2}{3 x-r-2}\right\}$, which strictly exceeds $\frac{x-1}{x}$, and in this case, contractual equilibrium selects a strict subset of the SPE Pareto frontier. ${ }^{13}$

Although none of the renegotiation refinements that GK studied allows for disagreement, in terms of their equilibrium construction, one can construe a refusal to make a specified transfer as a disagreement. To illustrate, consider a GK equilibrium that sustains $\mathrm{CC}$ along the equilibrium path. If player 1 refuses to make a transfer, he is first punished with DD in the subsequent action phase and then is required to make a transfer that yields him a zero payoff in the continuation. Since CC is still played in all future periods, the continuation payoff vector under disagreement is $(1-\delta)(0,2)$. If the players were able to bargain, their surplus in average terms would be $\delta 2>0$, and yet player 1 would capture none of it-as if he had no bargaining power. That is, reconciling this equilibrium with any notion of bargaining would require that all the bargaining power be endogenously reallocated to whichever player is not being punished. Section 8.1 further discusses history-dependent bargaining power.

To illustrate how bargaining power influences the contractual equilibrium set, consider the prisoners' dilemma when one player has all the bargaining

\footnotetext{
${ }^{12}$ Since $\underline{w}_{i}^{-i} \geq 0$ is required by individual rationality, $\pi_{i}>0$ implies $z_{i}^{-i}>0$.

${ }^{13}$ Another approach in the renegotiation-proofness literature allows Pareto-dominated punishments as long as the players recognize that such punishments are needed to support high payoffs at subsequent histories. For instance, the "consistent bargaining" theory of Abreu, Pearce, and Stacchetti (1993), which applies only to symmetric games, selects the best symmetric equilibrium from among those that maximize the minimum payoff any player earns at any history. Applying this notion prior to the transfer phase and, without loss of generality, employing stationary "simple strategies" as defined by GK, yields a straightforward conclusion: the value set of the optimal consistent bargaining equilibrium converges to $\{(1,1)\}$ as $\delta \rightarrow 1$.
} 


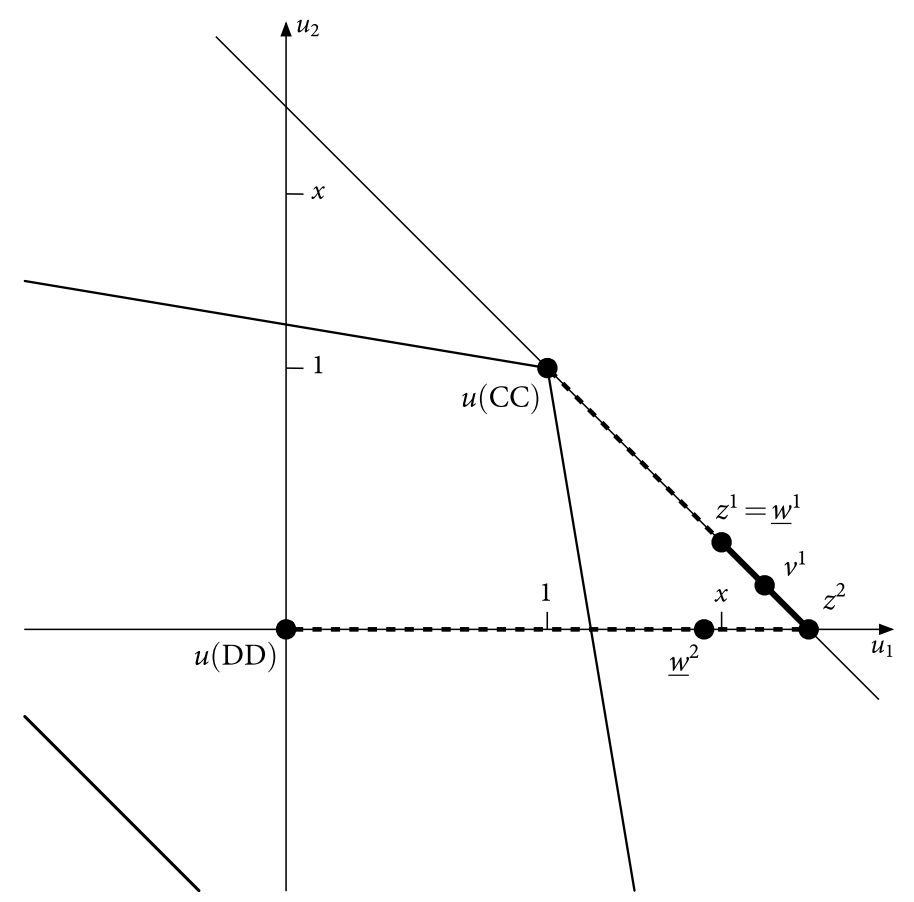

FIGURE 2.-The prisoners' dilemma with unequal bargaining power. The parameters satisfy $x<r, x<2$, and $\left(\pi_{1}, \pi_{2}\right)=(1,0)$. Each endpoint $z^{i}$ is attained by playing CC in the stage game and using a transfer to give player 1 all the surplus relative to the disagreement point $\underline{w}^{i}$. The disagreement point $\underline{w}^{2}$ is attained by playing DD in the stage game and continuing with promised utility $z^{2}$ regardless of whether a deviation occurs. The disagreement point $\underline{w}^{1}$ is attained by playing CC in the stage game and continuing with promised utility $v^{1}$ if no deviation occurs, but promised utility $z^{i}$ if player $i$ deviates unilaterally.

power, as illustrated in Figure 2. Suppose that $x-r<0$ and $x<2$; under these conditions, if both players had equal bargaining power, then in contractual equilibrium they could attain only $(0,0)$. But suppose instead that $\pi_{1}=1=1-\pi_{2}$. Then $\Gamma(d)=\gamma^{1}(d)=2-x$ whenever $\frac{\delta}{1-\delta} d \geq 2(x-1)$, because $\gamma^{2}(d)=0$ for all $d \geq 0$. State 2-which punishes player 2-uses DD under disagreement. ${ }^{14}$ Since player 2 has no bargaining power, she receives a payoff of zero under both agreement and disagreement. State 1 uses CC under both disagreement and agreement. This dramatically different construction arises because CC and DD are relatively cheap in terms of incentives (because $x<r$,

${ }^{14}$ Strictly speaking, there is an indeterminacy in state 2 behavior under disagreement-the $\arg \max$ of $\gamma^{2}$ includes both DC and DD when $\pi_{2}=0$. However, only DD arises along any sequence with $\pi_{2}>0$ and $\pi_{2} \rightarrow 0$. 
$\mathrm{CD}$ and DC are very expensive), and their projections onto the Pareto frontier in the direction of the bargaining power are widely separated.

These results show that contractual equilibrium generates specific predictions for the prisoners' dilemma, including predictions that distinguish it from renegotiation proofness.

\section{GENERALIZATION}

This section extends our analysis to a general model with more than two players, imperfect public monitoring, and heterogeneous discount factors. For simplicity, we do not specify the bargaining process, but instead assume that there exist bargaining weights that summarize the relevant backward induction solution of the bargaining phase under the axioms. (It would be cumbersome, but straightforward, to construct such a bargaining process using a generalized alternating-offer protocol. ${ }^{15}$ ) A simplified description of a game in this class is a tuple $\langle n, \mathcal{A}, \Theta, f, u, \boldsymbol{\delta}, \pi\rangle$, with the following components:

- A finite number of players $n$.

- A stage game, featuring a set of action profiles $\mathcal{A}=\mathcal{A}_{1} \times \cdots \times \mathcal{A}_{n}$, a set of public signals $\Theta$, a signal distribution function $f: \mathcal{A} \rightarrow \Delta \Theta$, and payoff functions $\left(u_{i}: \mathcal{A}_{i} \times \Theta \rightarrow \mathbb{R}\right)_{i=1}^{n}$.

- A vector of discount factors $\delta \in[0,1)^{n}$, where $\delta_{i}$ denotes player $i$ 's discount factor.

- Bargaining weights $\pi=\left(\pi_{1}, \pi_{2}, \ldots, \pi_{n}\right)$, with $\pi \geq 0$ and $\sum_{i=1}^{n} \pi_{i}=1$.

In the stage game, the players simultaneously select their actions, yielding action profile $a \in \mathcal{A}$. Public signal $\theta \in \Theta$ is then realized according to the probability measure $f(\cdot \mid a)$. The players publicly observe $\theta$, but they do not observe each other's actions. We write $u(a, \theta) \equiv\left(u_{i}\left(a_{i}, \theta\right)\right)_{i=1}^{n}$, and extend $f$ and $u$ to the space of mixed actions. We examine a "perfect public" notion of contractual equilibrium in which the disagreement point and all individual actions are conditioned on only the public history.

Since utility in average terms is no longer necessarily transferable, we express continuation payoffs in total terms rather than average terms. To make the difference clear in notation, values that were in average terms in previous sections but are now in total terms are shown with a tilde. The construction of a continuation value $\tilde{w}$ from the negotiation phase must now incorporate (i) continuation values as a function of the public signal and (ii) the players' possibly different discount factors. For any two vectors $x, x^{\prime} \in \mathbb{R}^{n}$, define the componentwise multiplication operator $*$ as $x * x^{\prime} \equiv\left(x_{1} x_{1}^{\prime}, x_{2} x_{2}^{\prime}, \ldots, x_{n} x_{n}^{\prime}\right)$. We use the standard notation $x \cdot x^{\prime}$ for the dot product of $x$ and $x^{\prime}$, and define 1 as

\footnotetext{
${ }^{15}$ Alternating-offer bargaining games with three or more players can have many equilibria. The multiplicity can be resolved by selecting the unique Markov-perfect equilibrium in the isolated bargaining game, which can be viewed as imposing NFD on each round of bargaining rather than merely after bargaining breaks down.
} 
the $n$-dimensional vector of ones. Then an agreement continuation value $\tilde{w}$ is constructed as

$$
\tilde{w}=m+\int_{\theta \in \Theta}(u(\alpha, \theta)+\boldsymbol{\delta} * \tilde{g}(\theta)) d f(\theta \mid \alpha),
$$

where $m \in \mathbb{R}_{0}^{n} \equiv\left\{m^{\prime} \in \mathbb{R}^{n} \mid \sum_{i=1}^{n} m_{i}^{\prime}=0\right\}$ is the transfer, $\alpha \in \hat{\Delta} \mathcal{A}$ is the mixed action profile, $\tilde{g}(\theta)$ is the continuation value from the start of the next period following public signal $\theta$, and $f(\theta \mid \alpha)$ is the probability measure on $\Theta$ that arises from $\alpha$. Similarly, a disagreement value is given by

$$
\underline{\tilde{w}}=\int_{\theta \in \Theta}(u(\alpha, \theta)+\boldsymbol{\delta} * \tilde{g}(\theta)) d f(\theta \mid \alpha) .
$$

We say that $\tilde{g}: \Theta \rightarrow \tilde{W}$ enforces $\alpha$ if $\alpha$ is a Nash equilibrium of the game with action-profile space $\mathcal{A}$ and payoffs given by $\int_{\theta \in \Theta}(u(a, \theta)+\boldsymbol{\delta} * \tilde{g}(\theta)) d f(\theta \mid a)$. Operators $D, C$, and $B$ are revised as

$$
\begin{aligned}
D(\tilde{W}) \equiv & \left\{\underline{\tilde{w}} \in \mathbb{R}^{n} \mid\right. \\
& \exists \tilde{g}: \Theta \rightarrow \tilde{W} \text { and } \alpha \in \hat{\Delta} \mathcal{A} \\
& \text { s.t. } \tilde{g} \text { enforces } \alpha \text { and Eq. (14) holds }\}, \\
C(\tilde{W}) \equiv & \left\{\tilde{w} \in \mathbb{R}^{n} \mid\right. \\
& \exists m \in \mathbb{R}_{0}^{2} \text { and } \tilde{w}^{\prime} \in D(W) \\
& \text { s.t. } \left.\tilde{w}=m+\tilde{w}^{\prime} \text { and } \tilde{w} \in \bar{D}(\tilde{W})\right\},
\end{aligned}
$$

where $\bar{D}$ is defined as before, and

$$
B(\tilde{W}, \underline{\tilde{W}}) \equiv\left\{\underline{\tilde{w}}+\pi\left(\max _{\tilde{w}^{\prime} \in \tilde{W}} \mathbf{1} \cdot \tilde{w}^{\prime}-\mathbf{1} \cdot \underline{\tilde{w}}\right) \mid \underline{\tilde{w}} \in \underline{\tilde{W}}\right\} .
$$

As before, $\tilde{W}$ is a BSG set if $\tilde{W}=\operatorname{co} B(C(\tilde{W}), D(\tilde{W}))$; the definition of full Pareto dominance carries over unchanged as well.

With $\mathcal{A}$ and $\Theta$ finite, we can guarantee existence.

THEOREM 8: Consider any n-player game in the simplified form $\langle n, \mathcal{A}, \Theta, f, u$, $\boldsymbol{\delta}, \pi\rangle$. If $\mathcal{A}$ and $\Theta$ are finite and $\delta_{i} \in[0,1)$ for all $i$, then the game has a unique dominant BSG set $\tilde{W}^{*}$. Moreover, $\tilde{W}^{*}$ is a compact hyperpolygon contained in a hyperplane normal to the vector $\mathbf{1}$.

The proof, given in Appendix B.2 in the Supplemental Material, examines a fixed-point problem for a transformation of the self-generation operator $\operatorname{co} B(C(\cdot), D(\cdot))$. The transformation normalizes the set of continuation values 
by subtracting the welfare level in a direction that accounts for the heterogeneous discount factors and bargaining shares. Since the normalized bargaining operator preserves compactness, and is bounded, monotone, and continuous on decreasing sets, Tarski's fixed-point theorem guarantees a largest fixed point-which is a hyperpolygon in $\mathbb{R}^{n}$.

For the special case of two players, $\tilde{V}\left(S_{\mathrm{CE}}\right)$ can be explicitly characterized even allowing for imperfect public monitoring and asymmetric discount factors $\boldsymbol{\delta}=\left(\delta_{1}, \delta_{2}\right)$. As in Section 5.1, the contractual equilibrium set is a compact line segment of slope -1 , where each endpoint is an agreement value formed with reference to an appropriately chosen disagreement point. Since the heterogeneous discount factors impose significant extra notation, we reserve the gory details for Appendix B.2.

For symmetric games, a small disparity in discount factors has a similar effect to a small disparity in bargaining power: as the following corollary shows, the more patient player gains and the less patient player loses, but in average terms, the gains outweigh the losses. Let $\bar{z}_{i} \equiv \frac{1}{2\left(1-\delta_{i}\right)}\left(z_{i}^{1}+z_{i}^{2}\right)$, that is, player $i$ 's average payoff across the two states, in average terms. Let $\bar{z}=\frac{1}{2}\left(\bar{z}_{1}+\bar{z}_{2}\right)$, that is, the average welfare across the two states, in average terms.

COROLLARY 2: If the stage game is symmetric and $\pi=\left(\frac{1}{2}, \frac{1}{2}\right)$, then, fixing the average discount factor, $\bar{z}$ strictly increases in any sufficiently small disparity between the discount factors, while the less patient player's average payoff across the two states strictly decreases.

\section{APPLICATION TO RELATIONAL CONTRACTING}

In this section, we use contractual equilibrium to examine the role of bargaining power in a principal-agent relationship and to explore the impact of external enforcement. We then comment on possible connections with the macro labor literature.

\subsection{A Principal-Agent Problem}

We first investigate the role of bargaining power in the context of a principalagent model with moral hazard, based on Levin (2003). We find that the agent's effort is increasing in his bargaining power and that the principal prefers an intermediate level of bargaining power.

In each stage game, the agent chooses effort $e \in[0, \bar{e}]$, incurring a cost $c(e)$, and then the principal makes a voluntary payment to the agent. The principal's revenue is a random variable $\theta$, with probability density $f(\cdot \mid e)$ and full support on a compact interval. The expected payoff vector in the stage game (i.e., excluding transfers) is $u(e)=\left(-c(e), \int \theta d f(\theta \mid e)\right)$. The principal does not observe $e$, but $\theta$ is public, so the principal's voluntary payment can be conditioned 
on $\theta$. We assume that $c$ is strictly increasing and strictly convex, $c(0)=0, f$ has the monotone likelihood property, $\int \theta d f(\theta \mid 0)=0$, and $f\left(\theta \mid e=c^{-1}(\cdot)\right)$ is convex. The players engage in bargaining and can make voluntary transfers before the stage game in each period, and they share a common discount factor $\delta<1$. We normalize payoffs by $(1-\delta)$ to put them in average terms.

Suppose there is no external enforcement. What is the maximum effort that can be sustained in equilibrium? The answer depends on the allocation of bargaining power, where $\pi_{\mathrm{A}} \geq 0$ is the agent's bargaining share and $1-\pi_{\mathrm{A}}$ is the principal's. Appendix B. 2 shows that in a two-player game such as this, it suffices to construct an equilibrium using a two-state machine, where state $\mathrm{P}$ rewards the agent and state A punishes the agent. The construction is illustrated in Figure 3.

In state $\mathrm{P}$, for disagreement play, we seek an effort level that rewards the agent-for example, zero effort. Since it is the agent's optimum in the stage game, there is no need for continuation utilities to vary so as to provide him incentives. So after a disagreement in state $\mathrm{P}$, the players stay in state $\mathrm{P}$

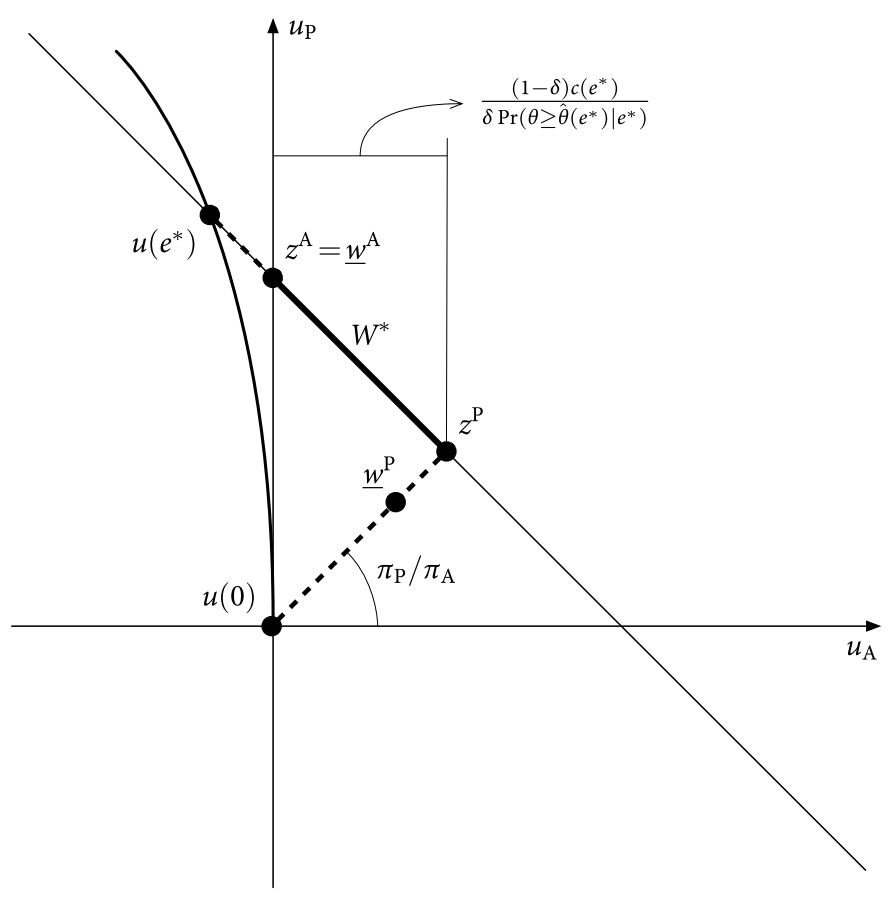

FIGURE 3.-The principal-agent game. The disagreement point $w^{\mathrm{P}}$ is attained by playing stage-game Nash equilibrium $e=0$ and continuing with promised utility $z^{\mathrm{P}}$ regardless of $\theta$. The disagreement point $\underline{w}^{\mathrm{A}}$ is attained by playing $e=e^{*}$ in the stage game, continuing with promised utility $z^{\mathrm{P}}$ if $\theta \geq \hat{\theta}\left(e^{*}\right)$, where $e^{*}$ is the maximum effort supported by the difference between $z_{\mathrm{A}}^{\mathrm{P}}$ and $z_{\mathrm{A}}^{\mathrm{A}}$, and continuing with promised utility $z^{\mathrm{A}}$ otherwise. 
the following period regardless of the realized output. Under agreement in state $\mathrm{P}$, the players recognize that their "outside option" is to disagree, implement zero effort, and then return to state $\mathrm{P}$ next period. Therefore, their utility (specifically, their vector of average discounted utilities) under disagreement is a convex combination of $(0,0)$ and their agreement utility in state P. Since the agent obtains a $\pi_{\mathrm{A}}$ fraction of the surplus, their agreement utility is $\left(\pi_{\mathrm{A}}, 1-\pi_{\mathrm{A}}\right) \cdot\left(\mathbb{E}\left(\theta \mid e^{*}\right)-c\left(e^{*}\right)\right)$, where $e^{*}$ is the equilibrium path effort. To attain this utility, the principal makes a payment to the agent as part of their agreement. The principal is willing to pay because doing so gives her strictly higher utility than does the disagreement that would arise should she fail to pay.

In state $\mathrm{A}$, we seek an effort level under disagreement that punishes the agent. The best candidate is the equilibrium path effort $e^{*}$, which is the highest effort that can be enforced using equilibrium continuation utilities. This effort level is enforced by staying in state A for low realizations of $\theta$ and transiting to state $\mathrm{P}$ for high realizations of $\theta \cdot{ }^{16}$ As Levin showed, the optimal cutoff for enforcing effort level $e^{*}$ is the output level $\hat{\theta}\left(e^{*}\right)$ at which $\left.\frac{\partial}{\partial e} f(\theta \mid e)\right|_{e=e^{*}}$, as a function of $\theta$, switches from negative to positive (by the monotone likelihood property, this identifies a unique output level). In fact, this same incentive scheme must be used whenever the agent is to exert effort $e^{*}$, that is, in state $\mathrm{P}$ under agreement as well as in state $\mathrm{A}$ under both agreement and disagreement.

Since optimal effort is played under disagreement in state A, disagreement is already on the Pareto frontier of what is attainable in equilibrium. Hence there is no surplus to share, so play is the same under agreement and disagreement. Because the agent can always deviate to zero effort in every period, his utility in state A must be at least zero. To provide the maximal incentives, in fact his utility in state A should be exactly zero.

Equilibrium path effort $e^{*}$ is thus a fixed point of the agent's optimization problem: ${ }^{17}$

$$
\begin{aligned}
e^{*} \in \underset{e \in[0, \bar{e}]}{\arg \max }(-(1-\delta) c(e) \\
\left.\quad+\delta \operatorname{Pr}\left(\theta \geq \hat{\theta}\left(e^{*}\right) \mid e\right) \pi_{\mathrm{A}}\left(\mathbb{E}\left(\theta \mid e^{*}\right)-c\left(e^{*}\right)\right)\right) .
\end{aligned}
$$

Because the principal and the agent negotiate over how to play, they will jointly select the highest fixed point. Since incentives are stronger the more weight the agent places on the second term in his objective function, we see that $e^{*}$ increases in $\delta$ and $\pi_{\mathrm{A}}$, and converges to efficient effort as $\delta \rightarrow 1$ and $\pi_{\mathrm{A}} \rightarrow 1$.

\footnotetext{
${ }^{16}$ If the principal could promise a voluntary payment for high realizations of $\theta$ in the action phase, it would have the same effect as transitioning to state $\mathrm{P}$.

${ }^{17} \mathrm{We}$ assume for convenience that $\bar{e} \in \arg \max _{e \in[0, \bar{e}]}[\mathbb{E}(\theta \mid e)-c(e)]$, so inefficiently high effort is infeasible.
} 
Observe that only zero effort is supported if the principal has all the bargaining power $\left(\pi_{\mathrm{A}}=0\right)$. Suppose that the agent exerts zero effort if the players have never agreed; then their agreement in the first period selects $z^{\mathrm{P}}$ as their initial payoffs. In that case, the principal also receives zero utility if she has no bargaining power $\left(\pi_{\mathrm{A}}=1\right)$, so her utility is nonmonotone in $\pi_{\mathrm{A}}$. An institution that selects a Pareto-optimal bargaining protocol will always endow the agent with some bargaining power, leading the agent to earn payoffs strictly higher than zero, that is, an internal efficiency wage. In particular, the principal will prefer to have more bargaining power for herself when it is easier to motivate the agent—such as when the discount factor is higher, when output is less noisy (holding the expected returns to effort fixed), or when there is an additional signal that is informative about the agent's effort.

Levin's "strongly optimal" equilibrium relies on continuation play that, following an out-of-equilibrium offer by the principal, punishes the principal if the agent rejected but punishes the agent if the agent accepted. Since the disagreement outcome is sensitive to the manner of disagreement, there is little role for the exercise of bargaining power. In contractual equilibrium, in contrast, NFD ensures a well-defined default point for bargaining each period, while internal and Pareto external agreement consistency allow the agents to endogenously select a division of the surplus that accords with their bargaining power.

\subsection{External Enforcement and Self-Enforcement}

To further illustrate the usefulness of contractual equilibrium, this section considers the question posed by Baker, Gibbons, and Murphy (1994; henceforth BGM): Does external enforcement help or harm self-enforcement? BGM focused on "trigger punishments" in which the agent punishes the principal for reneging on a voluntary payment by refusing to accept "implicit" (self-enforced) contracts offered in the future. When "explicit" (externally enforced) contracts are available, BGM assumed that even in a trigger punishment, the agent would accept an externally enforced contract and, therefore, the principal offers the externally enforced contract that maximizes her profit. ${ }^{18}$ BGM's key insight is that the threat of terminating the relationship is a more severe punishment than reverting to profit-maximizing externally enforced contracts and can, therefore, support higher payoffs in equilibrium. Thus, BGM found that externally enforced and self-enforced contracts can be substitutes.

\footnotetext{
${ }^{18} \mathrm{BGM}$ adopted this assumption "because the purely game-theoretic analyses of renegotiation abstract from institutions that would influence renegotiation in the labor market we consider." By explicitly modeling the bargaining process, our framework provides tools that answer this challenge.
} 
In contrast, recent empirical studies find complementarity between externally enforced and self-enforced contracts: better external enforcement improves the ability of partners to self-enforce their agreements (Beuve and Saussier (2012), Lazzarini, Miller, and Zenger (2004), Ryall and Sampson (2009)). We show here that complementarity follows generally from the analysis of contractual equilibrium.

A trigger punishment (either termination or reversion to only explicit contracts) is generally not viable under agreement in contractual equilibrium, because the principal and the agent can bargain their way out of it. Likewise, an explicit "spot market" contract of the sort considered by BGM would not arise under disagreement in contractual equilibrium, because it requires the agreement of both parties. That is, an externally enforced contract cannot serve as the players' "fallback position" unless it is already in place prior to bargaining. Thus, the fallback position is continued interaction under the parties' initial long-term contract (the employment contract).

To broaden our results, we generalize the model from Section 7.1 to allow for external enforcement of some less productive form of effort. At first, we take the externally enforced contract to be given exogenously; we discuss later how it might be chosen endogenously. We find that when the agent has some bargaining power, the availability of external enforcement always improves the prospects for self-enforcement. In particular, an optimal long-term externally enforced contract induces the agent to exert counterproductive effort by compensating him for his verifiable effort costs. This externally enforced contract is always undone by self-enforced incentives under agreement; it directly drives only behavior under disagreement. A surplus-destroying externally enforced contract raises the stakes of bargaining when the agent is rewarded, enabling the agent to capture more of the surplus. When the agent is being punished, the same behavior is implemented under disagreement and agreement, so there is no surplus to bargain over. Compared to the case without external enforcement, the difference in the agent's payoff under agreement in the two states is greater, supporting higher effort on the equilibrium path. ${ }^{19}$

The contractual equilibrium we describe is similar to that outlined in Section 7.1 and is illustrated in Figure 4. In state $\mathrm{P}$ under disagreement, the principal pays only to the letter of the externally enforced contract and the agent plays his stage-game best response. In state $\mathrm{A}$ under disagreement and in both states under agreement, the agent plays his equilibrium-path effort, and is optimally rewarded by transiting to state $\mathrm{P}$ for good outcomes and is punished by transiting to state A for bad outcomes.

Suppose that the agent chooses both the kind and the level of his effort. As before, his effort level is $e \in[0, \bar{e}]$ and his effort cost is $c(e)$. However, there

\footnotetext{
${ }^{19} \mathrm{Kval} \varnothing \mathrm{y}$ and Olsen (2009) found that relational contracting is strengthened when parties agree on a breach remedy that minimizes surplus. In their model, breach is triggered by a deviation in the action phase and leads to a trigger punishment in which bargaining is disallowed.
} 


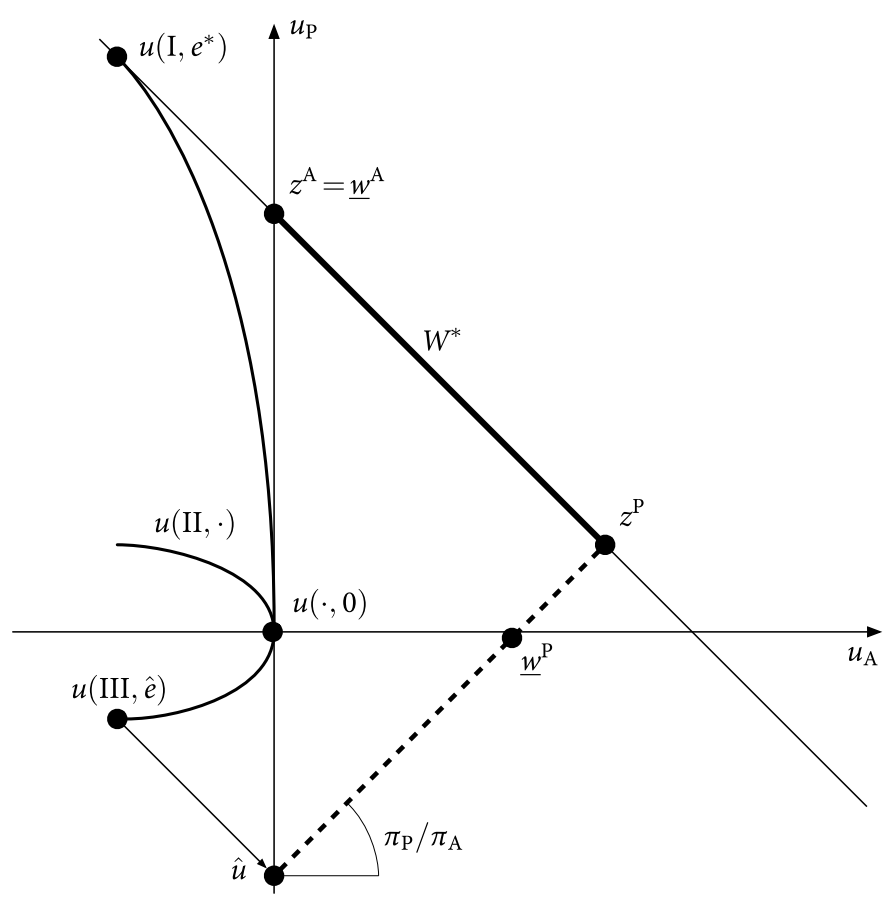

FIGURE 4.-The explicit contract game. Disagreement point $\underline{w}^{\mathrm{P}}$ is attained by playing the stage-game Nash equilibrium (III, $\hat{e}$ ) under the externally enforced contract, then continuing with promised utility $z^{\mathrm{P}}$ regardless of $\theta$. Disagreement point $\underline{w}^{\mathrm{A}}$ is attained by playing $\left(\mathrm{I}, e^{*}\right)$, continuing with promised utility $z^{\mathrm{P}}$ if $\theta \geq \hat{\theta}\left(\mathrm{I}, e^{*}\right)$ (where $e^{*}$ is the maximum type I effort supported by the payoff span of $W^{*}$ ) and continuing with promised utility $z^{\mathrm{A}}$ otherwise. Compared to the principal-agent game (see Figure 3), the payoff span of $W^{*}$ is larger, so higher effort is attained in equilibrium.

are now three kinds of effort. Type I effort is the same as in the previous section and is not externally enforceable. Type II effort is externally enforceable, but less productive: it generates distribution over output $\hat{f}(\cdot \mid e)$, satisfying the same conditions as $f$, but is first-order stochastically dominated by $f(\cdot \mid e)$ for every $e>0$. Type III effort is also externally enforceable, but is counterproductive: for concreteness and simplicity, assume that it generates a distribution $\tilde{f}(\cdot \mid e)$ such that $\tilde{f}(\theta \mid e)=\hat{f}(-\theta \mid e)$ for all $e$. Given the externally enforced contract, let $(\hat{E}, \hat{e})$ be the agent's stage-game best response, where $\hat{E} \in\{\mathrm{I}, \mathrm{II}, \mathrm{III}\}$, and let $\hat{u}$ be the expected stage-game payoff vector, including the externally enforced contractual payments, that results when the agent plays $(\hat{E}, \hat{e})$. Because the agent can always deviate to obtain $\hat{u}_{\mathrm{A}}$, his utility in each state must be at least $\hat{u}_{\mathrm{A}}$. To provide maximal incentives, of course, his utility in state $\mathrm{A}, z_{\mathrm{A}}^{\mathrm{A}}$, should be exactly $\hat{u}$ under both agreement and disagreement. 
In state $\mathrm{P}$ under agreement, the principal and the agent share the surplus according to their bargaining shares, so the agent's utility is

$$
\begin{aligned}
z_{\mathrm{A}}^{\mathrm{P}}= & (1-\delta) \hat{u}_{\mathrm{A}}+\delta z_{\mathrm{A}}^{\mathrm{P}}+\pi_{\mathrm{A}}\left(\int \theta d f\left(\theta \mid e^{*}\right)-c\left(e^{*}\right)\right. \\
& \left.-\left((1-\delta)\left(\hat{u}_{\mathrm{A}}+\hat{u}_{\mathrm{P}}\right)+\delta\left(z_{\mathrm{A}}^{\mathrm{P}}+z_{\mathrm{P}}^{\mathrm{P}}\right)\right)\right) \\
= & \hat{u}_{\mathrm{A}}+\pi_{\mathrm{A}}\left(\int \theta d f\left(\theta \mid e^{*}\right)-c\left(e^{*}\right)-\left(\hat{u}_{\mathrm{A}}+\hat{u}_{\mathrm{P}}\right)\right) .
\end{aligned}
$$

The strength of the agent's incentives is measured by the difference between his payoffs under agreement in state $\mathrm{P}$ and state $\mathrm{A}$; that is, $z_{\mathrm{A}}^{\mathrm{P}}-z_{\mathrm{A}}^{\mathrm{A}}=$ $\pi_{\mathrm{A}}\left(\mathbb{E}\left(\theta \mid e^{*}\right)-c\left(e^{*}\right)-\left(\hat{u}_{\mathrm{A}}+\hat{u}_{\mathrm{P}}\right)\right)$. Therefore, if $\pi_{\mathrm{A}}>0$, then his incentives are strictly decreasing in $\hat{u}_{\mathrm{A}}+\hat{u}_{\mathrm{P}}$. Intuitively, when joint payoffs are lower under disagreement in state $\mathrm{P}$, there is more to be gained from agreeing, and the agent gets a $\pi_{\mathrm{A}}$ share of the gains.

So far we have assumed that the externally enforced contract is exogenous. But suppose the externally enforced contract is jointly chosen by the principal and the agent in their first-period bargaining phase. Since their joint payoff in equilibrium is strictly decreasing in their joint payoff under disagreement in state P, they want their externally enforced contract to destroy enough surplus to support unconstrained efficient effort on the equilibrium path. In a broader environment, surplus destruction could be constrained by the ability of the principal to fire the agent at some cost and the agent to quit at some cost. If there are tight constraints on surplus destruction, then a straight salary without externally enforced incentives can be optimal.

\subsection{Connections With the Macro Labor Literature}

Bargaining power and moral hazard play important roles in the macro labor literature, but there is room to better integrate them and also allow for continued interaction under disagreement. On the role of bargaining power, the macro labor search literature (following Diamond and Maskin (1979)) examines long-term relationships in which trading partners meet in a frictional matching market and negotiate whether to initiate an employment relationship. Terms of trade are set according to the Nash bargaining solution, with the disagreement point being the parties' expected values of separating and returning to the unmatched pools. ${ }^{20}$ However, Hall and Milgrom (2008) have

\footnotetext{
${ }^{20}$ Mortensen and Pissarides (1994), den Haan, Ramey, and Watson (2000), and others extended this approach by applying the Nash bargaining solution at each instant to represent recurrent bargaining. den Haan, Ramey, and Watson (2003) and Genicot and Ray (2006) applied a similar approach to credit markets.
} 
criticized this assumption as unrealistic. Their point is that if separation is voluntary, then the disagreement point should be defined not as the parties' separation values, but as their continuation values in their current relationshipincluding the possibility, but not the requirement, of separation.

On moral hazard, workers can be motivated by "efficiency wages" that they would lose if fired from their jobs. Following Shapiro and Stiglitz (1984), traditional efficiency wage models assume that firms commit to a constant wage, so there is no ongoing bargaining between firms and workers. More recently, the relational contracts literature has started to address the question of how firms can commit endogenously to wage schedules that may vary with output or other monitoring signals. However, in these models, bargaining power either does not play a role (e.g., MacLeod and Malcomson (1989, 1998), Levin (2003)) or is applied only at the beginning of the relationship (e.g., Ramey and Watson (1997, 2001), Moen and Rosén (2011)).

Our analysis indicates that the institutional setting that underlies bargaining power may influence the returns to the employment relationship, because the worker's payoffs both on the equilibrium path and after a deviation (the difference between which constitutes the effective efficiency wage) are endogenously selected through the bargaining process. This is important not only when the moral hazard problem concerns the worker's efforts, but also where it concerns his investments, such as in human capital. ${ }^{21}$ It may be useful to empirically evaluate aspects of productive relationships that are influenced by bargaining power, and also find ways to measure bargaining power directly. Regarding the latter, at the industry or firm level, one might associate bargaining power with the level of unionization. For instance, Dobbelaere (2004) found that the total surplus divided between the firm and workers is positively correlated with unionization, which is consistent with our principal-agent example. In a related case study, Guy (2003) suggested that initiation of a customer service program increased the workers' bargaining power, resulting in better effort incentives, but also greater rents for the workers. Though these results may be suggestive, further research is needed to help understand the relationship between collective bargaining power at the union level and the moral hazard problems that play out primarily at the level of an individual worker.

\section{CONCEPTUAL DISCUSSION}

This section discusses links between our approach and the prior literatures on preplay communication and renegotiation proofness. We comment on the implications of varying the axioms and the structure of the model, and discuss connections with experimental work.

\footnotetext{
${ }^{21}$ In principle, these interrelationships could affect the propagation of shocks in macro labor models; see den Haan, Ramey, and Watson (2000) as well as Rogerson, Shimer, and Wright (2005) and Ramey (2011) for surveys of the literature.
} 


\subsection{Positive Foundations for Bargaining}

Our axioms have antecedents in the literature on preplay communication. Farrell $(1987,1988)$ allowed players to make cheap-talk announcements before choosing their payoff-relevant actions. Farrell looked at symmetric equilibria with the properties that (i) if the announced actions constitute an equilibrium in the second-stage subgame, then the players will select this action profile in the second stage; (ii) otherwise, the players will select an arbitrary equilibrium of the subgame. If one construes Farrell's condition (i) as "agreement" and condition (ii) as "disagreement," then our IAC and PEAC axioms relate to Farrell's first assumption, and our NFD axiom relates to his second assumption. Santos (2000) built on Farrell's approach by structuring the cheap-talk phase as a bargaining process and showed that inefficient Nash equilibria can arise if players condition their play in the subgame on the way in which disagreement occurs - a theme continued with our Theorem 1 . But only efficient Nash equilibria arise if the players do not condition on the history of messages in disagreement (a precursor of NFD). ${ }^{22}$

As in the preplay communication literature, the positive justification for our axioms is to allow the players to meaningfully discuss how to play. An axiomatic approach is necessary, because, as in any cheap-talk model, "babbling" is always a possibility; that is, the players could simply ignore all communication and bargaining would be meaningless. Accordingly, our axioms on the meaning of agreement-IAC and PEAC_-imply that players mean what they say when they propose and accept certain kinds of offers.

IAC and PEAC are weaker than renegotiation-proofness axioms in that they merely make these continuation equilibria available for the players to discuss, rather than require the players to accept them. Holding aside this difference, however, IAC and PEAC have direct counterparts in the renegotiationproofness literature. Specifically, IAC makes available for discussion any continuation play that is decomposable on the set of payoffs available at other histories in the same equilibrium, as contemplated by Ray's (1994) notion of "internal renegotiation proofness." It also relates closely to the "internal consistency" notion of Bernheim and Ray (1989) and the "weak renegotiation proofness" notion of Farrell and Maskin (1989). ${ }^{23}$ Similarly, PEAC makes available

\footnotetext{
${ }^{22}$ Watson (1991), Rabin (1994), and Arvan, Cabral, and Santos (1999) also built on Farrell's model. Watson (2006) provided a general treatment that embeds bargaining in finite-horizon games.

${ }^{23}$ Pearce (1987) critiqued these notions as follows: If the players find themselves in a punishment that is Pareto dominated by play at some other history, but the payoffs obtained at that other history are supported by the threat of a punishment similar to the one they currently face, then the idea that they could renegotiate to that other history is a contradiction. Internal consistency and related notions resolve the contradiction by invalidating the equilibrium, but Pearce (and, subsequently, Asheim (1991), Abreu, Pearce, and Stacchetti (1993), and Bergin and MacLeod (1993)) resolved the contradiction by invalidating the proposal. We do not mean to take a strong
} 
for discussion continuation play available in "better" equilibria, where one equilibrium is "better" than another if the value set of the first fully Pareto dominates the value set of the second. Since such equilibria are not vulnerable to Pareto-improving deviations back to the current equilibrium, PEAC's closest counterpart in the renegotiation-proofness literature is the "stationary Pareto perfection" notion of Asheim (1991).

A theory of disagreement is necessary because IAC and PEAC alone have no bite-it does not matter how players interpret agreements if there is no notion of how they should interpret disagreement. Our theory of disagreement, embodied in the NFD axiom, endows the bargaining phase with a well-defined disagreement point, which in turn allows the players' behavior to be interpreted in the context of the canonical alternating-offer bargaining paradigm. This paradigm is positively appealing because its backward induction solution is sensitive to both bargaining power (derived from the bargaining protocol) and the disagreement outcome. The backward induction solution also has normative foundations in static settings, since it is equivalent to the weighted Nash (1950) bargaining solution.

Still, it is not obvious that NFD is positively appealing in repeated settings: Would the other players not bear a grudge against, and single out for punishment, a player who deviates by offering to take a larger share for himself? We are sympathetic toward this critique. But the critique alone does not identify what the equilibrium offer should be in the first place. In fact, the critique leaves open the possibility that even a deviant offer that strictly Pareto dominates the equilibrium offer could engender a grudge and thereby be discouraged. A more finely tuned "theory of grudges" would indicate what kinds of deviant offers should engender grudges when bargaining breaks down. Depending on the details, such a theory might generate testable predictions that could distinguish it from NFD.

Our theory of disagreement could be modified to accommodate a limited notion of grudges without affecting our results. What is important about NFD is that no offer on the Pareto frontier of either $V(s)$ or $V\left(S_{\mathrm{CE}}\right)$ should engender a grudge if it also Pareto dominates the disagreement point. If the bargaining phase outcome is to be summarized using the Nash bargaining solution, a responder considering such an offer must evaluate it with respect to the same disagreement point that would obtain if he rejected the equilibrium offer. Other offers can engender grudges without disrupting the equilibrium bargaining outcome.

Other notions of disagreement are also potentially fruitful. The renegotiation-proofness literature, although it does not address the possibility of dis-

philosophical stand on this question. Indeed, imposing merely NFD and a variant of PEAC, without IAC, would yield a coherent theory of equilibrium selection, but one that is beyond the scope of this paper. 
agreement ${ }^{24}$ could be interpreted as imposing the theory that play under disagreement is identical to that under agreement. An alternative way to reconcile renegotiation proofness with our framework is to impose NFD and one or both consistency axioms, but allow the players' relative bargaining power to depend on the history. Allowing the bargaining weights to vary in arbitrary ways would enable the players to reach agreement on any point on the Pareto frontier of $C(W)$ as long as it Pareto dominated the disagreement point. For instance, the results of Levin (2003, discussed in Section 7.1) could be reconciled with our axioms if the agent got to make the take-it-or-leave-it offer in his reward state, but the principal got to make the offer in the agent's punishment state. However, it is our view that bargaining power should be derived from primitives of the model rather than treated as an aspect of equilibrium selection.

For symmetric games, Abreu, Pearce, and Stacchetti (1993) proposed a notion of "consistent bargaining equilibrium" that maximizes the minimum continuation payoff that any player can receive after any history, rather than imposing internal consistency. The bargaining process that generates their theory can be loosely conceptualized as follows. Each player has veto power over continuation play; if player $i$ exercises his veto, then they revert to a symmetric equilibrium of his choice, but starting from whichever history yields player $i$ 's worst continuation payoff in that equilibrium. This notion violates NFD, since continuation play following a veto depends on who exercised the veto. ${ }^{25}$ Abreu, Pearce, and Stacchetti interpreted veto power as a kind of equal bargaining power. They cautioned, however, that their notion should not be viewed as a "definitive solution," and challenged the literature to generate an "authoritative bargaining theory for the division of surplus in repeated games." Contractual equilibrium is our answer to this challenge.

\subsection{The Timing of Transfers and Bargaining}

In our modeling approach, the players bargain and then make voluntary transfers at the beginning of each period, and, by the NFD axiom, coordinate to make no transfers under disagreement. One way to interpret these assumptions is that, in reality, the monetary transfer marks the successful resolution of bargaining: If the agreement involves an immediate transfer-one player writing a check payable to the other-then it is the signature on the check, rather than the verbal "yes," that signifies the successful resolution of bargaining. Fail-

\footnotetext{
${ }^{24}$ One exception is Blume (1994), who allowed the players to agree to a plan that involves Pareto-dominated continuation values in the future. Blume's approach gives the players surplus to bargain over, but does not model bargaining power or investigate its implications. In principle, Blume's formal structure leaves open the possibility of modeling bargaining power by imposing axioms on the bargaining operator he defined.

${ }^{25}$ It is unclear how to resolve coordination problems that would arise when either multiple players wish to veto or each player would prefer that other players exercise their vetoes.
} 
ing to write the check implies disagreement. In this sense, the bargaining phase encompasses the transfer choices. ${ }^{26}$

Still, it could be interesting to consider variations on the timing of transfers and bargaining within a period. In the renegotiation-proofness literature, Baliga and Evans (2000) studied transfers that occur simultaneously with actions, while GK and Fong and Surti (2009) studied transfers that occur in a separate phase. In the context of contractual equilibrium, consider what could happen after a player reneges on an agreed-upon transfer. If there is no bargaining between the transfer and action phases, the players' continuation value may be any point in $D(W)$, the set of payoffs attainable from the action phase. In contrast, if there is an additional bargaining phase before the action phase, then the players should agree to continuation play on the Pareto frontier of $D(W)$. Depending on the stage game and the discount factor, this additional constraint may or may not restrict the set of attainable payoffs, as shown by GK.

Finally, what if there were no transfer phase between the bargaining and action phases, for example, if all transfers occurred simultaneously with actions? Contractual equilibrium would still constitute a coherent theory of equilibrium selection, but one would have to specify whether disagreement implies any restriction on how players coordinate their play in the action phase (such as no transfers). Without such a restriction, the $C$ and $D$ operators would be identical, so given any contractual equilibrium, one could always modify play under disagreement to be identical to play under agreement. There would be no surplus to bargain over, so bargaining power would play no role. The implications of contractual equilibrium would be similar to those of "consistent" equilibrium (Bernheim and Ray (1989)). In our model, surplus arises from the fact that the Pareto frontier is higher under agreement than under disagreement. For the study of relational contracts, we argue that transfers and productive actions are most appropriately modeled in separate phases, and that forcing them to occur simultaneously constitutes a knife-edge special case.

\subsection{Experimental Connections}

The experimental setting presents a good opportunity to develop intuition and test the predictions of contractual equilibrium against other theories. We are not aware of experiments on recurrent bargaining in infinitely repeated games. However, some experimental work on preplay communication and coordination relates to key elements of our theory.

Experiments on preplay communication suggest that players use messages to coordinate their behavior, and that the protocol by which they communi-

\footnotetext{
${ }^{26}$ Checks require a minimal degree of external enforcement. Our approach readily generalizes to settings in which other types of contractual clauses may also be externally enforceable.
} 
cate imparts a measure of bargaining power. For example, Cooper, DeJong, Forsythe, and Ross $(1989,1992)$ compared games with one-way and two-way communication. With one-way communication, the sender typically announces his preferred equilibrium of the underlying game and the players typically play this equilibrium. With simultaneous two-way communication, outcomes are more symmetric, but the players often fail to coordinate. Allowing multiple rounds of simultaneous messages reduces the incidence of coordination failure. $^{27}$

Cooper and Kühn (2013) provided provocative evidence on communication, coordination, and renegotiation in games. Cooper and Kühn conducted twoperiod experiments and studied the effect of allowing or disallowing communication between periods. Cooperation in the first period is more frequent when a player has threatened to use second-period play to punish a cheater (by coordinating on a Pareto-dominated equilibrium), suggesting that players use preplay communication to coordinate. Moreover, when players can communicate between periods, they often renegotiate away from punishments to select a Pareto-dominant equilibrium in the second period, as would be predicted by both our theory and renegotiation proofness. Nonetheless, cooperation in the first period is even higher with between-period communication. Players take the opportunity between periods to admonish one another (sometimes aggressively) for cheating in the first period. Cooper and Kühn interpreted these admonishments as nonpecuniary punishments that may be less costly to the admonisher than punishing via actions in the second-period stage game.

Clear distinctions between contractual equilibrium and renegotiation proofness do not arise in the results of Cooper and Kühn, because their experimental design does not allow for monetary transfers, variations in relative bargaining power, or variations in stage-game payoffs. ${ }^{28}$ However, we can suggest a related experiment to test the distinct predictions of contractual equilibrium. We propose an experiment with the following features: (i) the players can make voluntary transfers between the two periods; (ii) the second-period coordination game has a Pareto-dominant Nash equilibrium, but also has asymmetric Pareto-dominated equilibria; (iii) communication between periods follows a specific bargaining protocol. ${ }^{29}$ Varying the equilibrium payoffs of the coordination game would produce different disagreement values and surpluses. By

\footnotetext{
${ }^{27}$ See Crawford (1998) for a description of this and other cheap-talk experiments.

${ }^{28}$ Sloof and Sonnemans (2011) studied the Schmidt and Schnitzer model with communication and transfers. However, the experimental design focuses on technology choice rather than bargaining, and so does not separate the Schmidt and Schnitzer model from standard subgameperfection analysis.

${ }^{29}$ Players might engage in less verbal admonishment when they have access to monetary transfers as an even cheaper punishment instrument, in addition to multiple equilibria to use as disagreement points.
} 
varying the bargaining protocol, one could look at different degrees of relative bargaining power. Contractual equilibrium predicts that these variations should matter for supporting cooperation in the first period. It may also be useful to have noisy play in the first period, so that it is not known whether deviations from an agreement were intentional.

\section{CONCLUSION}

This paper introduces contractual equilibrium, a new approach to equilibrium selection in repeated games that is based on communication with endogenous meaning. Contractual equilibrium allows disagreement play and bargaining power to arise endogenously, in a way consistent with a well-defined extensive form. Contractual equilibrium exists, yields a unique welfare level, is tractable in applications, and extends easily to games with imperfect monitoring and heterogeneous discount factors.

The underlying principles of contractual equilibrium are not restricted to repeated games with transfers. In dynamic games, where a payoff-relevant state can vary over time and shift endogenously in response to players' actions, the same notions of agreement and disagreement apply. Endogenous disagreement can be particularly powerful in a dynamic setting, since the actions taken under disagreement can change the set of feasible continuation values. ${ }^{30} \mathrm{Fi}$ nally, it would be interesting to consider how disagreement play might depend on which coalitions manage to cohere when the grand coalition fails to agree. We view all of these extensions as promising areas for further research.

Since the noncooperative solution of the generalized alternating-offer bargaining game under our axioms is expressed in terms of bargaining power, it yields the same outcome as the generalized Nash bargaining solution. Hence the same conclusions would arise from a hybrid model in which the noncooperative bargaining and transfer phases are replaced with a cooperative Nash bargaining phase. ${ }^{31}$

\footnotetext{
${ }^{30}$ The legal environment- the set of enforceable contracts and the enforcement technologycan introduce dynamics if long-term contracts are enforceable. Then the players' signatures on the contract, though they have no effect on the feasibility of the equilibrium path since the contract can always be renegotiated, can leverage the threat of enforcement to change the feasible set of continuation values after future disagreements.

${ }^{31}$ There is a tradition of incorporating cooperative bargaining into otherwise noncooperative games to model bargaining power. One example is the macroeconomic literature on labor market search frictions, as discussed in Section 7.3. Another is the holdup theory of the firm originated by Grossman and Hart (1986) and Hart and Moore (1990). Schmidt and Schnitzer (1995) and Ramey and Watson (2002) employed a hybrid approach to relational contracts. Harrington (1989) and Brandenburger and Stuart (2007) examined the actions that take place leading up to an agreement, where the agreement yields a contract that terminates the game.
} 


\section{APPENDIX A: PROOFS}

\section{A.1. No-Bite Theorems}

We expand Theorem 1 by invoking a stronger consistency axiom while proving the same result. Since the players recognize that any payoff in $C\left(\operatorname{co} V\left(S_{\text {IAC }}\right)\right)$ is attainable from the action phase, suppose that any proposal to play as if switching to a different equilibrium in $S_{\text {IAC }}$ is interpreted meaningfully.

AXIOM SEAC-Strong External Agreement Consistency: For every history to the transfer phase under agreement, if the agreement takes the form $w \in$ $C\left(\operatorname{co} V\left(S_{\mathrm{IAC}}\right)\right)$, then continuation play yields the value $w$.

Let $S_{\mathrm{SEAC}}$ be the subset of $S_{\mathrm{SPE}}$ that satisfies SEAC and note that $S_{\mathrm{SEAC}} \subseteq$ $S_{\text {IAC }}$. SEAC imposes a strong requirement on continuation play following a deviant agreement, because it requires the players to follow through even on a deviant agreement that is Pareto dominated by what they should have agreed on. However, IAC and SEAC do not constrain the set of attainable payoffs.

\section{THEOREM 1'-IAC and SEAC Have No Bite: We have}

$$
V\left(S_{\mathrm{IAC}}\right)=V\left(S_{\mathrm{SEAC}}\right)=V\left(S_{\mathrm{SPE}}\right) .
$$

PROOF: Let $S_{\mathrm{NB}}$ be the set of equilibria in a repeated game that lacks any bargaining phase, but is otherwise identical to the game described in Section 2.1. Since bargaining is cheap talk and both games have arbitrary public correlation devices, $V\left(S_{\mathrm{SPE}}\right)=V\left(S_{\mathrm{NB}}\right)$. Thus, our task is to prove $V\left(S_{\mathrm{NB}}\right) \subseteq$ $V\left(S_{\text {SEAC }}\right)$. We proceed by demonstrating how, for a given period, values in $C\left(V\left(S_{\mathrm{NB}}\right)\right)$ can be supported from the bargaining phase in a way that is consistent with SEAC. We then construct equilibrium strategies recursively.

Consider any $w \in C\left(V\left(S_{\mathrm{NB}}\right)\right)$. By the definition of $C$, there is a specification of behavior for the transfer phase and action phase that (i) yields value $w$ from the transfer phase, (ii) utilizes continuation values from $V\left(S_{\mathrm{NB}}\right)$ for the following period, and (iii) is sequentially rational. We refer below to this specification as "rational behavior supporting $w$ from the transfer phase."

For any fixed value in $C\left(V\left(S_{\mathrm{NB}}\right)\right)$, we next describe behavior in the bargaining phase that yields this value. We specify the behavior recursively by round of the bargaining phase, starting with the first round. Take as given a round of the bargaining phase and any $w \in C\left(V\left(S_{\mathrm{NB}}\right)\right)$. Call $w$ the desired value. Let $i$ denote the proposer and let $j$ denote the responder. The following provisions give the prescribed behavior for this round of bargaining.

(i) Prescribe that player $i$ proposes $w$ and player $i$ responds "yes." If the players behave in this way, then prescribe rational behavior supporting $w$ from the transfer phase. 
(ii) Suppose player $i$ proposes $w$ as prescribed, but player $j$ deviates by responding "no." If bargaining then breaks down, prescribe rational behavior supporting $w$ from the transfer phase. Otherwise, let $w$ again be the desired value for the next round of bargaining.

(iii) Suppose player $i$ deviates by proposing some $w^{\prime} \notin C\left(V\left(S_{\mathrm{NB}}\right)\right)$. If bargaining terminates after player $j$ 's response, either because player $j$ responds "yes" or because bargaining breaks down, then prescribe rational behavior supporting $w$ from the transfer phase. If bargaining does not terminate, then let $w$ be the desired value for the next round of bargaining.

(iv) Suppose player $i$ deviates by proposing some $w^{\prime} \in C\left(V\left(S_{\mathrm{NB}}\right)\right)$. Then select $w^{\prime \prime} \in C\left(V\left(S_{\mathrm{NB}}\right)\right)$ such that $w_{i}^{\prime \prime} \leq w_{i}$ and $w_{j}^{\prime \prime} \geq w_{j}^{\prime}$. (By the characterization of $C$ given by Eq. (3), $w^{\prime \prime}$ can always be attained by augmenting either $w$ or $w^{\prime}$ with a transfer from player $i$ to player $j$.) Prescribe that player $j$ responds "no" to the offer $w^{\prime}$. If player $j$ instead deviates to respond "yes," then prescribe rational behavior supporting $w^{\prime}$ from the transfer phase. If player $j$ responds "no" and bargaining breaks down, then prescribe rational behavior supporting $w^{\prime \prime}$ from the transfer phase. If player $j$ responds "no" and bargaining continues, then set the desired value to $w^{\prime \prime}$ for the next round of bargaining.

In some of the cases just described, bargaining continues to another round. Behavior for the next round is specified in the same fashion, with the new desired value. In this way, we recursively construct a complete specification of behavior in the bargaining phase, as well as for the transfer phase and the action phase. There is an implied mapping from the sequence of actions in the period to continuation values from the start of the next period.

The specified behavior is sequentially rational. For instance, by proposing the desired value $w$, player $i$ expects to get $w_{i}$. If he deviates, then he gets either $w_{i}$ or some $w_{i}^{\prime \prime} \leq w_{i}$, so he prefers to propose $w$. Note that in the event that player $i$ proposes some $w^{\prime} \in C\left(V\left(S_{\mathrm{NB}}\right)\right)$ (even a value satisfying $w_{j}^{\prime}>w_{j}$ ), player $j$ optimally says "no" because this leads to the value $w^{\prime \prime}$ which player $j$ prefers to $w^{\prime}$.

The specified behavior is also consistent with SEAC. To see this, first note that the presence of the public randomization phase implies co $V\left(S_{\mathrm{NB}}\right)=$ $V\left(S_{\mathrm{NB}}\right)$. In addition, since $S_{\mathrm{IAC}} \subseteq S_{\mathrm{NB}}$, we have that $\operatorname{co} V\left(S_{\mathrm{IAC}}\right) \subseteq \operatorname{co} V\left(S_{\mathrm{NB}}\right)$. Putting these together and using the fact that $C$ is monotone, we conclude that $C\left(\operatorname{co} V\left(S_{\mathrm{IAC}}\right)\right) \subseteq C\left(V\left(S_{\mathrm{NB}}\right)\right)$. Thus, any agreement on a value in $C\left(\operatorname{co} V\left(S_{\mathrm{IAC}}\right)\right)$ is covered by item (i) or item (iv), which specify behavior that attains the agreed value.

The construction above shows that all values in $C\left(V\left(S_{\mathrm{NB}}\right)\right)$ can be supported from the bargaining phase in a way consistent with SEAC, using continuation values in $V\left(S_{\mathrm{NB}}\right)$ from the following period. Thus, SEAC does not impose a constraint on the recursive construction that characterizes SPE continuation values, and $V\left(S_{\mathrm{SEAC}}\right)=V\left(S_{\mathrm{NB}}\right)=V\left(S_{\mathrm{SPE}}\right)$. For each point $v \in V\left(S_{\mathrm{SPE}}\right)$, an equilibrium strategy profile that attains value $v$ and satisfies SEAC can be constructed recursively by following the steps outlined above for behavior 
within a period and then over all periods (by tracking the continuation values required).

ProOF OF THEOREM 2: For the first claim of the theorem, we proceed by characterizing a subset $\hat{W} \subset V\left(S_{\mathrm{NFD}}^{\mathrm{ps}}\right)$ defined by the additional restriction that, if a player offers the disagreement value and the other player accepts, then this value is achieved in the continuation (IAC applied only to $\underline{w}$ ). A variant of operator $C$ can be used to characterize $\hat{W}$ :

$$
\begin{aligned}
\hat{C}(W) \equiv & \left\{w \in \mathbb{R}^{2} \mid\right. \\
& \exists m \in \mathbb{R}_{0}^{2} \text { and } \underline{w}, w^{\prime} \in D(W) \\
& \text { s.t. } \left.w=(1-\delta) m+w^{\prime} \text { and } w \geq \underline{w}\right\} .
\end{aligned}
$$

Note that $\hat{C}(W) \subseteq C(W)$ because $D(W) \subseteq \bar{D}(W)$

Given that continuation values in $W$ are available in the future, a payoff $w$ is available in the present if there exists a transfer $m$, a disagreement point $\underline{w} \in D(W)$, and an action-phase continuation value $w^{\prime} \in D(W)$ such that $w=(1-\delta) m+w^{\prime}$ and $w \geq \underline{w}$. This works because in this class of equilibria, each player $i$ can guarantee himself a payoff of at least $\underline{w}_{i}$ by always offering $\underline{w}$ as the proposer and always saying "no" as the responder. If $w$ Pareto dominates $\underline{w}$, then the transfer is incentive compatible because it makes both players better off than under disagreement. Since $w^{\prime} \in D(W)$, the actions are incentive compatible as well.

Standard arguments establish that the sets of interest are compact, and public randomization allows us to focus on convex sets. Let $W \subset \mathbb{R}^{2}$ be any compact and convex set. Operators $C$ and $\hat{C}$ share a useful property: The Pareto frontiers of $C(W)$ and $\hat{C}(W)$ are subsets of a line segment with slope -1 . In fact, they have the same extremal values. To see this, suppose that $w$ is the point that minimizes player 1's value among points in $P(C(W)$ ), so that $w_{1}=\min \bar{D}_{1}(W)$. There is then a point $\underline{w} \in D(W)$ such that $\underline{w}_{1}=w_{1}$. Furthermore, it must be that $\underline{w}_{2} \leq w_{2}$, for otherwise $w$ would not be on the Pareto frontier of $C(W)$. This implies that $w \in \hat{C}(W)$ as well. The same logic applies to the other extremal point. Since $\hat{C}(W) \subset C(W)$, the extrema coincide. We thus have that $P(\operatorname{co} C(W))=P(\operatorname{co} \hat{C}(W))$. It is also clear that each player's greatest and least values in $\operatorname{co} C(W)$ and $\operatorname{co} \hat{C}(W)$ are found on the Pareto frontiers of these sets.

With these facts in mind, we can restrict attention to sets $W$ that have the same properties, since our objects of interest are the fixed points of $\operatorname{co} C(\cdot)$ and co $\hat{C}(\cdot)$. With pure strategies, for any history to the action phase, the players will be coordinating on a pure action profile. It is sufficient to punish a unilateral deviation by selecting a continuation value that is worst for the deviating player. Furthermore, to find the maximal joint continuation value from 
the action phase, on the equilibrium path, the continuation value from the next period will be on the Pareto frontier of the set of continuation values. Thus, if $W$ is the set of continuation values from the next period, then, in reference to Eq. (1), it is sufficient to have $g$ map to three points on the frontier line segment, including the two endpoints. An implication of these observations is that $P(\operatorname{co} C(W))$ and $P(\operatorname{co} \hat{C}(W))$ depend only on the Pareto frontier of $W$. Thus, $P\left(V\left(S_{\mathrm{SPE}}^{\mathrm{ps}}\right)\right)$ is itself a fixed point of $\operatorname{co} C(\cdot)$ and also of co $\hat{C}(\cdot)$, which implies the first claim of the theorem.

For the second claim, observe that any subgame-perfect equilibrium value obtained without transfers can be obtained under NFD if the players ignore the bargaining phase entirely.

Q.E.D.

\section{A.2. Representation}

Theorem 3 is proven by the following five lemmas.

LEMMA 6: Consider an isolated noncooperative bargaining game with recognition process $\rho$, breakdown process $\beta$, default payoff $\underline{w}$, and bargaining set $\left\{w \in \mathbb{R}^{2} \mid w_{1}+w_{2} \leq K\right\}$ for some $k \in \mathbb{R}$ satisfying $K \geq \underline{w}_{1}+\underline{w}_{2}$. In any subgameperfect equilibrium, with probability 1 , each player i earns a payoff of $\underline{w}_{i}+\pi_{i}(K-$ $\left.\underline{w}_{1}-\underline{w}_{2}\right)$, where $\pi_{i} \equiv \sum_{\ell=1}^{\infty} \rho_{i, \ell} \beta_{\ell} \prod_{k=1}^{\ell-1}\left(1-\beta_{k}\right)$.

The proof follows from generalizing the recursive methods of Shaked and Sutton (1984), using our assumption that $\prod_{\ell=1}^{\infty}\left(1-\beta_{\ell}\right)=0$.

For the rest of the proof, fix $\pi_{i}=\sum_{\ell=1}^{\infty} \rho_{i, \ell} \beta_{\ell} \prod_{k=1}^{\ell-1}\left(1-\beta_{k}\right)$. Given a BSG set, we next construct a subgame-perfect equilibrium that attains a value in this set while satisfying IAC and NFD.

LEMMA 7: Let $W$ be a BSG set and select any payoff vector $v \in W$. Inputting $(v, W)$ into the recursive construction algorithm in Figure 5 yields a subgameperfect equilibrium $s \in S_{\mathrm{IAC}} \cap S_{\mathrm{NFD}}$ that attains an expected payoff of $v$.

PROOF: Note that the algorithm recursively constructs function $\hat{v}: \mathcal{H} \rightarrow W$ along with the strategy profile $s$, starting with the null history, then oneperiod histories, and so on. In line 5 of Figure $5, \mu$ exists because $v \in$ co $B(C(W), D(W)$ ), which follows from $W$ being a BSG set. In line 7, $\underline{w}$ exists by definition of $B$. In line 8, bargaining ends in agreement on payoff $w$ by construction of $w$ and Lemma 6 . In line $11, m^{*}$ and $w^{*}$ exist by definition of $C$. In line 19, if the test condition is satisfied for $i=j$, then it is violated for $i=-j$. In line 20, $w^{*}$ exists because $w^{\prime} \in C(W)$ satisfies $w^{\prime} \in \bar{D}(W)$ by definition of $C$. In line 21 , since $w^{*} \in D(W), \alpha^{*}$ and $g^{*}$ exist by definition of $D$. Therefore, the algorithm is well-defined. 
Input: $W$ a BSG set and $v \in W$.

Output: $s \in S_{\mathrm{IAC}} \cap S_{\mathrm{NFD}}$ such that $v \in V(s)$.

INITIALIZATION:

let $\hat{v}(\varnothing)=v$;

let $\Omega=B(C(W), D(W))$;

for $t=1$ to $\infty$ do

foreach $h \in\left(\Omega \times \mathcal{B} \times \mathbb{R}_{+} \times \mathbb{R}_{+} \times \mathcal{A}\right)^{t-1}$ do

PUBLIC RANDOMIZATION:

let $\mu \in \Delta \Omega$ solve $\int_{w \in \Omega} w d \mu=\hat{v}(h)$;

play public randomization $\mu$, resulting in realization $w$;

BARGAINING PHASE:

let $\underline{w} \in D(W)$ solve $w \in B(C(W),\{\underline{w}\})$;

play a subgame perfect equilibrium in an isolated noncooperative bargaining game with recognition process $\rho$, breakdown process $\beta$, bargaining set $C(W)$, and default payoff $\underline{w}$;

TRANSFER PHASE:

\section{foreach $b \in \mathcal{B}$ do}

if $b$ ends in agreement on a payoff $w^{\prime} \in C(W)$ then

else

let $\left(m^{*}, w^{*}\right) \in \mathbb{R}_{0}^{2} \times D(W)$ solve $(1-\delta) m^{*}+w^{*}=w^{\prime} ;$

let $m^{*}=(0,0)$ and $w^{*}=\underline{w}$;

let $\hat{m}^{*}=\left(-\min \left\{0, m_{1}^{*}\right\},-\min \left\{0, m_{2}^{*}\right\}\right)$;

play transfer $\hat{m}^{*}$;

ACTION PHASE:

foreach $\hat{m} \in \mathbb{R}_{+}^{2}$ do

if $b$ ends in agreement on a payoff $w^{\prime} \in C(W)$ then

foreach $i \in\{1,2\}$ do

if $\hat{m}_{i}<\hat{m}_{i}^{*}$ and $\hat{m}_{-i}=\hat{m}_{-i}^{*}$ then

19

let $w^{*} \in D(W)$ solve $w_{i}^{*} \leq w_{i}^{\prime}$;

21

let $\alpha^{*} \in \hat{\Delta} \mathcal{A}$ and $g^{*}: \mathcal{A} \rightarrow W$ solve $(1-\delta) u\left(\alpha^{*}\right)+\delta g^{*}\left(\alpha^{*}\right)=w^{*}$ subject to $\alpha^{*}$ is a Nash equilibrium in the game

$\left\langle\mathcal{A},(1-\delta) u+\delta g^{*}\right\rangle$;

play mixed action $\alpha^{*}$;

foreach $a \in \mathcal{A}$ do

let $\hat{v}(h, \mu, b, \hat{m}, a)=g^{*}(a)$;

FIGURE 5.-Recursive construction algorithm. 
Collecting the output of all the play statements yields a well-defined strategy profile (where line 6 is a move of Nature), since every history is accounted for. By construction, this equilibrium is subgame-perfect, since it calls for sequentially rational actions in every bargaining phase (by Lemma 6), sequentially rational transfers in every transfer phase (by lines 10-13 and 16-20), and sequentially rational mixed actions in every action phase (by line 21).

Axiom NFD is satisfied by line 13, which sets all disagreements to yield the same disagreement payoff $\underline{w}$. Axiom IAC is satisfied by lines 10-11 and 17-20, which ensure that any agreement payoff $w^{\prime} \in C(W)$ with $w^{\prime} \in \bar{D}(W)$ is implemented. Therefore, if $W$ is a BSG set and $v \in W$, the output is a strategy profile $s \in S_{\mathrm{IAC}} \cap S_{\mathrm{NFD}}$. The equilibrium payoff is $v \in V(s)$ by construction. Q.E.D.

LEMMA 8: If $s \in S_{\mathrm{IAC}} \cap S_{\mathrm{NFD}}$, then $\operatorname{co} V(s)$ is a BSG set.

PROOF: Since $s \in S_{\mathrm{NFD}}$, every bargaining phase has a well-defined disagreement payoff. Since $s \in S_{\text {IAC }}$, every proposal $w \in C(\operatorname{co} V(s))$, if accepted, leads to continuation payoff $w$. Standard recursive techniques for characterizing equilibrium continuation values in bargaining games (for instance, relating the proposer's supremum values in one round to the players' supremum continuation values from the next round) establish that the bargaining phase has a unique equilibrium outcome and that it has the same representation as in Lemma 6. Further, co $V(s)$ must have a closed Pareto boundary; otherwise existence is violated in the bargaining phase. By sequential rationality, the disagreement payoff $\underline{w}$ after any history satisfies $\underline{w} \in D(V(s)) \subseteq$ $D(\operatorname{co} V(s))$. Thus the equilibrium payoff from the bargaining phase is $w \in$ $B(C(\operatorname{co} V(s)), D(\operatorname{co} V(s)))$, so every continuation value from the start of a period satisfies $v \in \operatorname{co} B(C(\operatorname{co} V(s)), D(\operatorname{co} V(s)))$. Hence $\operatorname{co} V(s)$ is a BSG set.

Q.E.D.

For the following two lemmas, let $W^{*}$ be the dominant BSG set.

LEMMA 9: Inputting $W^{*}$ and any $v \in W^{*}$ into the recursive construction algorithm of Figure 5 yields a strategy profile $s^{*} \in S_{\text {PEAC }}$ such that $v \in V\left(s^{*}\right)$.

ProOF: By definition, $W^{*}$ fully Pareto dominates every BSG set. By Lemma 8 , therefore, $W^{*}$ fully Pareto dominates $V(s)$ for every equilibrium $s \in S_{\mathrm{IAC}} \cap S_{\mathrm{NFD}}$. Since $W^{*}$ is also a BSG set, every $v \in W^{*}$ is available in each bargaining phase. Since the recursive construction algorithm always produces an equilibrium $s \in S_{\mathrm{IAC}} \cap S_{\mathrm{NFD}}$, with input $W^{*}$ it produces an equilibrium $s^{*} \in S_{\text {PEAC }}$.

Q.E.D.

LEMMA 10: If $s^{*} \in S_{\text {PEAC }}$, then $V\left(s^{*}\right) \subseteq W^{*}$. 
ProOF: By Lemma 8, $\operatorname{co} V\left(s^{*}\right)$ is a BSG set. By PEAC and Lemma 9, every payoff in $W^{*}$ is in the bargaining set of every bargaining phase. By sequential rationality, therefore, no $v \in V\left(s^{*}\right)$ can be strictly Pareto dominated by any $v^{\prime} \in W^{*}$. This implies that if $W^{*}$ fully Pareto dominates $V\left(s^{*}\right)$, then it must be that $V\left(s^{*}\right) \subseteq W^{*}$. Finally, since $W^{*}=\operatorname{co} W^{*}$, we have co $V\left(s^{*}\right) \subseteq W^{*}$. Q.E.D.

\section{A.3. Existence and Construction}

PROOF OF LEMMA 1: By construction of $B$, for any set $W$, the elements of $B(C(W), D(W))$ are on a line of slope -1 , so $\operatorname{co} B(C(W), D(W))$ is a line segment of slope -1 . That $W \subseteq B(C(W), D(W))$ implies that $W \subseteq V\left(S_{\mathrm{SPE}}\right)$, wherein each $v \in V\left(S_{\mathrm{SPE}}\right)$ is bounded above by $v_{1}+v_{2} \leq$ $\max _{\alpha \in \hat{\Delta} \mathcal{A}}\left(u_{1}(\alpha)+u_{2}(\alpha)\right)$ and bounded below by $v_{i} \geq \min _{\alpha_{-i} \in \Delta \mathcal{A}_{-i}} \max _{\alpha_{i} \in \Delta \mathcal{A}_{i}} u_{i}(\alpha)$ for each $i$.

Q.E.D.

The following lemma establishes some basic properties of $B, C$, and $D$. Elsewhere we take these properties for granted without reference to this lemma.

LEMMA 11: (i) $W$ nonempty $\Rightarrow D(W)$ nonempty $\Rightarrow C$ ( $W$ ) nonempty. (ii) $W$ compact $\Rightarrow D(W)$ compact $\Rightarrow C(W)$ closed. (iii) $P(W)$ a nonempty line segment of slope -1 and $\underline{W}$ nonempty with $P(W \cup \underline{W})=P(W) \Rightarrow B(W, \underline{W})$ nonempty; (iv) $\underline{W}$ also compact $\Rightarrow B(W, \underline{W})$ compact $\Rightarrow \operatorname{co} B(W, \underline{W})$ compact.

The results in Lemma 11 follow from the definitions of $B, C$, and $D$, the fact that the Nash equilibrium correspondence is upper hemicontinuous, and the fact that every stage-game Nash equilibrium is enforced by a constant continuation value function.

Proof of Lemma 2: Since $W$ is a closed BSG set and $z^{2}$ is the endpoint that most favors player $1, z_{1}^{2}$ is the maximum payoff for player 1 that can be supported utilizing continuation values from $W$ associated with the next period, that is, $z_{1}^{2}=\max \left\{v_{1} \mid v \in B(C(W), D(W))\right\}$. Because elements in $B(C(W), D(W))$ correspond to various disagreement points in $D(W)$, this maximization problem can be expressed using the bargaining solution (Eq. (4) with $C(W)$ in place of $W)$, taking the disagreement point as the choice variable. Since level $(W)=\max _{w \in C(W)}\left(w_{1}+w_{2}\right)$ for any given disagreement point $\underline{w} \in D(W)$, in bargaining, player 1 earns the value $\underline{w}_{1}+\pi_{1}\left(\operatorname{level}(W)-\underline{w}_{1}-\underline{w}_{2}\right)$. Therefore,

$$
\begin{aligned}
& z_{1}^{2}=\max _{\underline{w}, g, \alpha}\left(\pi_{2} \underline{w}_{1}-\pi_{1} \underline{w}_{2}+\pi_{1} \operatorname{level}(W)\right) \\
& \text { s.t. } \quad \underline{w}=(1-\delta) u(\alpha)+\delta g(\alpha) \text { and } g: \mathcal{A} \rightarrow W \text { enforces } \alpha .
\end{aligned}
$$

We next rewrite the optimization problem with a change of variables. Define $\eta(a) \equiv g_{1}(a)-z_{1}^{2}$ for every $a$. Because the welfare level of every point in $W$ is level $(W)$, we have $g_{2}(a)=z_{2}^{2}-\eta(a)$. Also, the constraint that $g(a) \in \operatorname{co}\left\{z^{1}, z^{2}\right\}$ is equivalent to the requirement that $\eta(a) \in\left[z_{1}^{1}-z_{1}^{2}, 0\right]$. Using $\eta$ to substitute 
for $g$ and $\underline{w}$, and combining terms, we see that Eq. (21) is equivalent to

$$
\begin{aligned}
& z_{1}^{2}=\max _{\eta, \alpha}\left((1-\delta)\left(\pi_{2} u_{1}(\alpha)-\pi_{1} u_{2}(\alpha)\right)\right. \\
& \left.+\delta\left(\pi_{2} z_{1}^{2}-\pi_{1} z_{2}^{2}\right)+\delta \eta(\alpha)+\pi_{1} \operatorname{level}(W)\right) \\
& \text { s.t. } \quad\left\{\begin{array}{c}
\eta: \mathcal{A} \rightarrow\left[z_{1}^{1}-z_{1}^{2}, 0\right] \text { extended to } \hat{\Delta} \mathcal{A} \\
\alpha \in \hat{\Delta} \mathcal{A} \text { is a Nash equilibrium } \\
\text { of }\left\langle\mathcal{A},(1-\delta) u+\delta(\eta,-\eta)+\delta z^{2}\right\rangle
\end{array}\right.
\end{aligned}
$$

In the objective function on the right, we substitute for $z_{2}^{2}$ using the equality $z_{1}^{2}+z_{2}^{2}=\operatorname{level}(W)$. Combining terms and moving the constant $\delta z_{1}^{2}$ to the left side yields

$$
z_{1}^{2}=\pi_{1} \operatorname{level}(W)+\max _{\eta, \alpha}\left(\pi_{2} u_{1}(\alpha)-\pi_{1} u_{2}(\alpha)+\frac{\delta}{1-\delta} \eta(\alpha)\right),
$$

subject to the conditions above. Recalling the definition of $\gamma^{2}$, we have the conclusion of the lemma. Analyzing endpoint $z^{1}$ in the same way gives the similar expression for $z_{2}^{1}$.

The optimum defining $\gamma^{i}$ exists for each $i$ because the stage game is finite, the set of feasible $\eta$ functions is compact, and the Nash correspondence is upper hemicontinuous. Regarding the final claim of the lemma, let $\alpha^{\mathrm{NE}}$ be a Nash equilibrium of the stage game. Note that $\alpha^{\mathrm{NE}}$ and $\eta \equiv 0$ satisfy the conditions of the maximization problems that define $\gamma^{1}$ and $\gamma^{2}$, so $\gamma^{2}(d) \geq \pi_{2} u_{1}\left(\alpha^{\mathrm{NE}}\right)-\pi_{1} u_{2}\left(\alpha^{\mathrm{NE}}\right)$ and $\gamma^{1}(d) \geq \pi_{1} u_{2}\left(\alpha^{\mathrm{NE}}\right)-\pi_{2} u_{1}\left(\alpha^{\mathrm{NE}}\right)$. Summing, we have that $\gamma^{2}(d)+\gamma^{1}(d) \geq 0$.

Q.E.D.

ProOf OF Lemma 3: Let $z^{1}$ and $z^{2}$ denote the endpoints of $W$, and let $z^{1}$ and $z^{\prime 2}$ denote the endpoints of $W^{\prime}$. Suppose that $\operatorname{span}(W) \geq \operatorname{span}\left(W^{\prime}\right)$. The larger span of $W$ can support weakly more mixed actions in the stage game as equilibria than $W^{\prime}$ can, because it provides a greater range of continuation values. This comparison does not depend on the location of the endpoints or the levels of the two sets (which are mere constants in the players' payoffs), only their relative spans. Thus any mixed action that can be supported by $W^{\prime}$ can also be supported by $W$, so level $(W) \geq \operatorname{level}\left(W^{\prime}\right)$. Furthermore, since each $\gamma^{j}(d)$ is increasing in $d$, Lemma 2 and the larger span of $W$ imply that $z_{i}^{j} \geq z_{i}^{\prime j}$ for each $i \neq j$, which suffices to establish that $W$ fully Pareto dominates $W^{\prime}$.

Q.E.D.

\section{REFERENCES}

Abreu, D., D. G. Pearce, And E. Stacchetti (1990): “Toward a Theory of Discounted Repeated Games With Imperfect Monitoring," Econometrica, 58 (5), 1041-1063. [2305,2309] 
(1993): "Renegotiation and Symmetry in Repeated Games," Journal of Economic Theory, 60, 217-240. [2321,2333,2335]

Arvan, L., L. M. B. CABral, AND V. SAntos (1999): "Meaningful Cheap Talk Must Improve Equilibrium Payoffs,” Mathematical Social Sciences, 37 (1), 97-106. [2333]

AshEIM, G. B. (1991): "Extending Renegotiation-Proofness to Infinite Horizon Games," Games and Economic Behavior, 3 (3), 278-294. [2333,2334]

BAKer, G., R. GibBons, And K. J. Murphy (1994): "Subjective Performance Measures in Optimal Incentive Contracts," Quarterly Journal of Economics, 109 (4), 1125-1156. [2306, 2328]

(2002): "Relational Contracts and the Theory of the Firm," Quarterly Journal of Economics, 117 (1), 39-84. [2306]

BALIGA, S., AND R. Evans (2000): "Renegotiation in Repeated Games With Side-Payments," Games and Economic Behavior, 33, 159-176. [2336]

BERGIN, J., AND W. B. MACLEOD (1993): "Efficiency and Renegotiation in Repeated Games," Journal of Economic Theory, 61 (1), 42-73. [2333]

BERnheIM, B. D., AND D. RAY (1989): "Collective Dynamic Consistency in Repeated Games," Games and Economic Behavior, 1 (4), 295-326. [2307,2333,2336]

BEUVE, J., AND S. SAUSSIER (2012): "Interfirm Cooperation in Strategic Relationships: The Role of Formal Contract," Industrial and Corporate Change, 21 (4), 811-836. [2329]

BINMORE, K. (1987): "Perfect Equilibria in Bargaining Models," in The Economics of Bargaining, ed. by K. Binmore and P. Dasgupta. Oxford: Basil Blackwell, Chapter 5, 77-105. [2312]

Blume, A. (1994): "Intraplay Communication in Repeated Games," Games and Economic Behavior, 6 (2), 181-211. [2335]

BRANDEnBurger, A., AND H. Stuart (2007): “Biform Games,” Management Science, 53 (4), 537-549. [2338]

Chassang, S. (2010): "Building Routines: Learning, Cooperation, and the Dynamics of Incomplete Relational Contracts," American Economic Review, 100 (1), 448-465. [2306]

COOPER, D. J., AND K.-U. KÜHN (2013): "Communication Renegotiation, and the Scope for Collusion," American Economic Journal: Microeconomics (forthcoming). [2337]

CoOper, R., D. V. DeJong, R. Forsythe, AND T. W. Ross (1989): "Communication in the Battle of the Sexes Game: Some Experimental Results," RAND Journal of Economics, 20 (4), 568-587. [2337]

(1992): "Communication in Coordination Games," Quarterly Journal of Economics, 107 (2), 739-771. [2337]

CRAWFORD, V. (1998): "A Survey of Experiments on Communication via Cheap Talk," Journal of Economic Theory, 78 (2), 286-298. [2337]

DEN HAAN, W. J., G. RAMEY, AND J. WATSON (2000): "Job Destruction and Propagation of Shocks," American Economic Review, 90 (3), 482-498. [2331,2332]

(2003): "Liquidity Flows and Fragility of Business Enterprises," Journal of Monetary Economics, 50, 1215-1241. [2331]

DiAMOND, P. A., AND E. S. MASKIN (1979): "An Equilibrium Analysis of Search and Breach of Contract, I: Steady States," The Bell Journal of Economics, 10 (1), 282-316. [2331]

Dobbelaere, S. (2004): "Estimation of Price-Cost Margins and Union Bargaining Power for Belgian Manufacturing," International Journal of Industrial Organization, 22 (10), 1381-1398. [2332]

DOORNIK, K. (2006): "Relational Contracting in Partnerships," Journal of Economics and Management Strategy, 15 (2), 517-548. [2306]

FARRELL, J. (1987): “Cheap Talk, Coordination, and Entry,” RAND Journal of Economics, 18 (1), 34-39. [2333]

(1988): "Communication, Coordination and Nash Equilibrium," Economics Letters, 27 (3), 209-214. [2333]

FARRELL, J., AND E. S. MASKIN (1989): "Renegotiation in Repeated Games," Games and Economic Behavior, 1 (4), 327-360. [2307,2321,2333] 
FONG, Y.-F., AND J. LI (2010): “Information Revelation in Relational Contracts,” Working Paper, available at http://goo.gl/BfmMm. [2306]

(2012): "Relational Contracts, Efficiency Wages, and Employment Dynamics,” Working Paper, available at http://goo.gl/0367r. [2306]

FONG, Y.-F., AND J. SURTI (2009): "The Optimal Degree of Cooperation in the Repeated Prisoners' Dilemma With Side Payments," Games and Economic Behavior, 67 (1), 277-291. [2336]

FuCHS, W. (2007): "Contracting With Repeated Moral Hazard and Private Evaluations," American Economic Review, 97 (4), 1432-1448. [2306]

FudENBERG, D., AND E. S. MASKIN (1986): "The Folk Theorem in Repeated Games With Discounting or With Incomplete Information," Econometrica, 54 (3), 533-554. [2307]

GENICOT, G., AND D. RAY (2006): "Bargaining Power and Enforcement in Credit Markets," Journal of Development Economics, 79, 398-412. [2331]

GOLDLÜCKE, S., AND S. KRANZ (2012): "Infinitely Repeated Games With Public Monitoring and Monetary Transfers," Journal of Economic Theory, 147 (3), 1191-1221. [2310]

(2013): "Renegotiation-Proof Relational Contracts," Games and Economic Behavior, 80, 157-178. [2321]

Grossman, S., AND O. HART (1986): "The Costs and Benefits of Ownership: A Theory of Lateral and Vertical Integration,” Journal of Political Economy, 94, 691-719. [2338]

GUY, F. (2003): "High-Involvement Work Practices and Employee Bargaining Power," Employee Relations, 25 (5), 455-471. [2332]

HALAC, M. (2012): "Relational Contracts and the Value of Relationships," American Economic Review, 102 (2), 750-779. [2306]

HALL, R. E., AND P. R. MiLgROM (2008): “The Limited Influence of Unemployment on the Wage Bargain,” American Economic Review, 98 (4), 1653-1674. [2331]

HARRINGTON, JR., J. E. (1989): "Collusion Among Asymmetric Firms: The Case of Different Discount Factors," International Journal of Game Theory, 7 (2), 289-307. [2338]

HART, O., AND J. MOORE (1990): "Property Rights and the Nature of the Firm," Journal of Political Economy, 98, 1119-1158. [2338]

Hermalin, B. E., L. LI, AND T. NAUGHTON (2013): "The Relational Underpinnings of Formal Contracting and the Welfare Consequences of Legal System Improvement," Economics Letters, 119 (1), 72-76. [2306]

JACKSON, M. O., AND T. R. PALFREY (1998): "Efficiency and Voluntary Implementation in Markets With Repeated Pairwise Bargaining," Econometrica, 66 (6), 1353-1388. [2306]

KLIMENKO, M., G. RAMEY, AND J. WATSON (2008): "Recurrent Trade Agreements and the Value of External Enforcement," Journal of International Economics, 74 (2), 475-499. [2306]

KVALøY, O., AND T. E. OLSEN (2006): "Team Incentives in Relational Employment Contracts," Journal of Labor Economics, 24 (1), 139-169. [2306]

(2009): "Endogenous Verifiability and Relational Contracting," American Economic Review, 99 (5), 2193-2208. [2306,2329]

LaZZARini, S. G., G. J. Miller, AND T. R. Zenger (2004): “Order With Some Law: Complementarity versus Substitution of Formal and Informal Arrangements," Journal of Law, Economics, and Organization, 20 (2), 261-298. [2329]

LEVIN, J. (2002): "Multilateral Contracting and the Employment Relationship," Quarterly Journal of Economics, 117 (3), 1075-1103. [2306]

(2003): "Relational Incentive Contracts," American Economic Review, 93 (3), 835-857. [2306,2321,2325,2327,2328,2332,2335]

MACLEOD, W. B., AND J. M. MALCOMSON (1989): "Implicit Contracts, Incentive Compatibility, and Involuntary Unemployment," Econometrica, 57 (2), 447-480. [2306,2332]

2332]

(1998): "Motivation and Markets," American Economic Review, 88 (3), 388-411. [2306,

MilleR, D. A., AND J. WATSON (2013): "Supplement to 'A Theory of Disagreement in Repeated Games With Bargaining,", Econometrica Supplemental Material, 81, http://www. econometricsociety.org/ecta/supmat/10361_proofs.pdf. [2318] 
Moen, E. R., AND Å. Rosén (2011): “Incentives in Competitive Search Equilibrium,” Review of Economic Studies, 78 (2), 733-761. [2332]

Mortensen, D. T., AND C. A. Pissarides (1994): "Job Creation and Job Destruction in the Theory of Unemployment," Review of Economic Studies, 61 (3), 397-415. [2331]

NASH, J. (1950): “The Bargaining Problem,” Econometrica, 18 (2), 155-162. [2315,2334]

PEARCE, D. G. (1987): "Renegotiation-Proof Equilibria: Collective Rationality and Intertemporal Cooperation," Working Paper, available at http://goo.gl/2O585. [2307,2333]

PEARCE, D. G., AND E. STACCHETTI (1998): “The Interaction of Implicit and Explicit Contracts in Repeated Agency," Games and Economic Behavior, 23, 75-96. [2306]

RABIN, M. (1994): “A Model of Pre-Game Communication,” Journal of Economic Theory, 63 (2), 370-391. [2333]

RADNER, R. (1985): "Repeated Principal-Agent Games With Discounting," Econometrica, 53 (5), 1173-1198. [2306]

RAFF, H., AND D. SCHMIDT (2000): “Cumbersome Coordination in Repeated Games," International Journal of Game Theory, 29 (1), 101-118. [2306]

RAMEY, V. (2011): "A Brief History of the Real Business Cycle-Search \& Matching Literature," Manuscript, available at http://goo.gl/FOsA6. [2332]

RAMEY, G., AND J. WATSON (1997): "Contractual Fragility, Job Destruction, and Business Cycles," Quarterly Journal of Economics, 112 (3), 873-911. [2306,2332]

(2001): "Bilateral Trade and Opportunism in a Matching Market," Contributions to Theoretical Economics, 1 (1). [2306,2332]

(2002): "Contractual Intermediaries," Journal of Law, Economics, and Organization, 18 (2), 362-384. [2306,2338]

RAY, D. (1994): "Internally Renegotiation-Proof Equilibrium Sets: Limit Behavior With Low Discounting," Games and Economic Behavior, 6 (1), 162-177. [2333]

RAYO, L. (2007): "Relational Incentives and Moral Hazard in Teams," Review of Economic Studies, 74 (3), 937-963. [2306]

Rogerson, R., R. SHIMER, AND R. Wright (2005): "Search-Theoretic Models of the Labor Market: A Survey," Journal of Economic Literature, 43 (4), 959-988. [2332]

Rubinstein, A. (1980): "Strong Perfect Equilibrium in Supergames," International Journal of Game Theory, 9, 1-12. [2307,2321] [2312]

(1982): "Perfect Equilibrium in a Bargaining Model," Econometrica, 50 (1), 97-109.

RYALL, M. D., AND R. C. SAMPSON (2009): "Formal Contracts in the Presence of Relational Enforcement Mechanisms: Evidence From Technology Development Projects," Management Science, 55 (6), 906-925. [2329]

SANTOS, V. (2000): "Alternating-Announcements Cheap Talk," Journal of Economic Behavior \& Organization, 42 (3), 405-416. [2333]

SCHMIDT, K. M., AND M. SCHNITZER (1995): "The Interaction of Explicit and Implicit Contracts," Economics Letters, 48 (2), 193-199. [2306,2337,2338]

SHAKED, A., AND J. SUTTON (1984): "Involuntary Unemployment as a Perfect Equilibrium in a Bargaining Model," Econometrica, 52 (6), 1351-1364. [2312,2342]

ShAPIRO, C., AND J. E. STiglitz (1984): "Equilibrium Unemployment as a Worker Discipline Device," American Economic Review, 74 (3), 433-444. [2332]

SLOOF, R., AND J. SonNEMANS (2011): "The Interaction Between Explicit and Relational Incentives: An Experiment," Games and Economic Behavior, 73 (2), 573-594. [2337]

ThOmas, J., AND T. WORRALl (1988): "Self-Enforcing Wage Contracts," Review of Economic Studies, 55 (4), 541-553. [2306]

WATSON, J. (1991): "Communication and Superior Cooperation in Two-Player Normal Form Games,” Economics Letters, 35 (3), 267-271. [2333]

- (2006): "Contract Game Theory: Basic Concepts for Settings With Finite Horizons," Working Paper, available at http://goo.gl/NFtle. [2333] 
Dept. of Economics, University of Michigan, 611 Tappan St., Ann Arbor, MI 48109, U.S.A.; econdm@umich.edu

$$
\text { and }
$$

Dept. of Economics, University of California, San Diego, 9500 Gilman Dr., La Jolla, CA 92093-0508, U.S.A.; jwatson@ucsd.edu.

Manuscript received October, 2011; final revision received March, 2013. 